\title{
POPOVICIU'S TYPE INEQUALITIES FOR $h$-MN-CONVEX FUNCTIONS
}

\author{
MOHAMMAD W. ALOMARI
}

\begin{abstract}
In this work, several inequalities of Popoviciu type for $h$-MN-convex functions are proved, where $\mathrm{M}$ and $\mathrm{N}$ are denote to Arithmetic, Geometric and Harmonic means and $h$ is a non-negative superadditive or subadditive function.
\end{abstract}

\section{INTRODUCTION}

The class of $h$-convex functions, which generalizes convex, $s$-convex (denoted by $K_{s}^{2}$, [4]), Godunova-Levin functions (denoted by $Q(I)$, [7]) and $P$-functions (denoted by $P(I)$, [15]), was introduced by Varošanec in [18]. Namely, the $h$-convex function is defined as a non-negative function $f: I \rightarrow \mathbb{R}$ which satisfies

$$
f(t \alpha+(1-t) \beta) \leq h(t) f(\alpha)+h(1-t) f(\beta),
$$

where $h$ is a non-negative function, $t \in(0,1) \subseteq J$ and $x, y \in I$, where $I$ and $J$ are real intervals such that $(0,1) \subseteq J$. Accordingly, some properties of $h$-convex functions were discussed in the same work of Varošanec. The famous references about these classes are [5], [8] and [10].

In this work, $I$ and $J$ are two intervals subset of $(0, \infty)$ (unless we specified) such that $(0,1) \subseteq J$ and $[a, b] \subseteq I$, where $0<a<b$. Define the function $\mathrm{M}:[0,1] \rightarrow[a, b]$ given by $\mathrm{M}(t)=\mathrm{M}(t ; a, b)$; where by $\mathrm{M}(t ; a, b)$ we mean one of the following functions:

(1) $A_{t}(a, b):=(1-t) a+t b ; \quad$ The generalized Arithmetic Mean.

(2) $G_{t}(a, b)=a^{1-t} b^{t}$; The generalized Geometric Mean.

(3) $H_{t}(a, b):=\frac{a b}{t a+(1-t) b}=\frac{1}{A_{t}\left(\frac{1}{a}, \frac{1}{b}\right)} ; \quad$ The generalized Harmonic Mean.

Note that $\mathrm{M}(0 ; a, b)=a$ and $\mathrm{M}(1 ; a, b)=b$. Clearly, for $t=\frac{1}{2}$, the means $A_{\frac{1}{2}}, G_{\frac{1}{2}}$ and $H_{\frac{1}{2}}$, respectively; represents the midpoint of the $A_{t}, G_{t}$ and $H_{t}$, respectively.

Also, we note that the above means are related with celebrated inequality

$$
H_{t}(a, b) \leq G_{t}(a, b) \leq A_{t}(a, b), \quad \forall t \in[0,1] .
$$

Using fruitful structures of $\mathbb{R}$ as a vector space, Anderson et al. in [2] defined midconvex functions with respect to a another mathematical means, by replacing a given mean, such as $G_{\frac{1}{2}}$ and $H_{\frac{1}{2}}$; instead of the arithmetic mean $A_{\frac{1}{2}}$. Recently, Alomari [1] generalized Anderson et al. work by extendng the classes of MN-convexity to be such as:

Definition 1. Let $h: J \rightarrow(0, \infty)$ be a positive function. Let $f: I \rightarrow(0, \infty)$ be any function. Let $\mathrm{M}:[0,1] \rightarrow[a, b]$ and $\mathrm{N}:(0, \infty) \rightarrow(0, \infty)$ be any two Mean functions. We say $f$ is $h$-MN-convex (-concave) or that $f$ belongs to the class $\overline{\mathcal{M N}}(h, I)(\underline{\mathcal{M N}}(h, I))$ if

$$
f(\mathrm{M}(t ; x, y)) \leq(\geq) \mathrm{N}(h(t) ; f(x), f(y)),
$$

Date: October 28, 2017.

2000 Mathematics Subject Classification. 26D15, 26E60, 26A51.

Key words and phrases. $h$-convex function; means; popoviciu inequality. 
for all $x, y \in I$ and $t \in[0,1]$.

Obviously, if $\mathrm{M}(t ; x, y)=A_{t}(x, y)=\mathrm{N}(t ; x, y)$, then Definition 1 reduces to the original concept of $h$-convexity. Also, if we assume $f$ is continuous, $h(t)=t$ and $t=\frac{1}{2}$ in (1.1), then the Definition 1 reduces to the Anderson et al. definition in [2].

In the same work [1], the author extended the classes $Q(I), P(I)$ and $K_{s}^{2}$ by replacing the arithmetic mean by another given one, as follows:

(1) Let $s \in(0,1]$, a function $f: I \rightarrow(0, \infty)$ is $\mathrm{M}_{\mathrm{t}} \mathrm{N}_{\mathrm{t}}-s$-convex function or that $f$ belongs to the class $K_{s}^{2}\left(I ; \mathrm{M}_{\mathrm{t}}, \mathrm{N}_{\mathrm{t}}\right)$ if for all $x, y \in I$ and $t \in[0,1]$ we have

$$
f(\mathrm{M}(t ; x, y)) \leq \mathrm{N}\left(t^{s} ; f(x), f(y)\right) .
$$

(2) We say that $f: I \rightarrow(0, \infty)$ is an Extended Godunova-Levin function or that $f$ belongs to the class $Q\left(I ; \mathrm{M}_{\mathrm{t}}, \mathrm{N}_{\mathrm{t}}\right)$ if for all $x, y \in I$ and $t \in(0,1)$ we have

$$
f(\mathrm{M}(t ; x, y)) \leq \mathrm{N}\left(\frac{1}{t} ; f(x), f(y)\right) .
$$

(3) We say that $f: I \rightarrow(0, \infty)$ is $P-\mathrm{M}_{\mathrm{t}} \mathrm{N}_{\mathrm{t}}$-function or that $f$ belongs to the class $P\left(I ; \mathrm{M}_{\mathrm{t}}, \mathrm{N}_{\mathrm{t}}\right)$ if for all $x, y \in I$ and $t \in[0,1]$ we have

$$
f(\mathrm{M}(t ; x, y)) \leq \mathrm{N}(1 ; f(x), f(y)) \text {. }
$$

In (1.2)-(1.4), setting $\mathrm{M}(t ; x, y)=\mathrm{A}_{\mathrm{t}}(x, y)=\mathrm{N}(t ; x, y)$, we then refer to the original definitions of these classes.

Let $h$ be a non-negative function such that $h(t) \geq t$ for $t \in(0,1)$. For instance $h_{r}(t)=t^{r}$, $t \in(0,1)$ has that property. In particular, for $r \leq 1$, if $f$ is a non-negative $\mathrm{M}_{\mathrm{t}} \mathrm{N}_{\mathrm{t}}$-convex function on $I$, then for $x, y \in I, t \in(0,1)$ we have

$$
f(\mathrm{M}(t ; x, y)) \leq \mathrm{N}(t ; f(x), f(y)) \leq \mathrm{N}\left(t^{r} ; f(x), f(y)\right)=\mathrm{N}(h(t) ; f(x), f(y)),
$$

for all $r \leq 1$ and $t \in(0,1)$. So that $f$ is $h-\mathrm{M}_{\mathrm{t}} \mathrm{N}_{\mathrm{t}}$-convex. Similarly, if the function satisfies the property $h(t) \leq t$ for $t \in(0,1)$, then $f$ is a non-negative $h$ - $\mathrm{M}_{\mathrm{t}} \mathrm{N}_{\mathrm{t}}$-concave. In particular, for $r \geq 1$, the function $h_{r}(t)$ has that property for $t \in(0,1)$. So that if $f$ is a non-negative $\mathrm{M}_{\mathrm{t}} \mathrm{N}_{\mathrm{t}}$-concave function on $I$, then for $x, y \in I, t \in(0,1)$ we have

$$
f(\mathrm{M}(t ; x, y)) \geq \mathrm{N}(t ; f(x), f(y)) \geq \mathrm{N}\left(t^{r} ; f(x), f(y)\right)=\mathrm{N}(h(t) ; f(x), f(y)),
$$

for all $r \geq 1$ and $t \in(0,1)$, which means that $f$ is $h-\mathrm{M}_{\mathrm{t}} \mathrm{N}_{\mathrm{t}}$-concave.

After focus consideration we find that, there is neither nonnegative $\frac{1}{t}-\mathrm{M}_{\mathrm{t}} \mathrm{A}_{\mathrm{t}}$-concave nor $\frac{1}{t}$ $\mathrm{M}_{\mathrm{t}} \mathrm{H}_{\mathrm{t}}$-convex functions, where $M_{t}=A_{t}, G_{t}, H_{t}$. The same observation holds for $h(t)=t^{k}$, $k \leq-1, t \in(0,1)$.

To see how this holds, suppose on the contrary that there is a nonnegative function $f$ which is $\frac{1}{t}-\mathrm{M}_{\mathrm{t}} \mathrm{A}_{\mathrm{t}}$-concave on $I$. Thus, for Means $\mathrm{M}_{\mathrm{t}}$ and $\mathrm{A}_{\mathrm{t}}$, the reverse inequality of (1.3) holds for all all $x, y \in I$ and $t \in(0,1)$.

$$
f(M(t ; x, y)) \geq \frac{1}{1-t} f(x)+\frac{1}{t} f(y) .
$$

Since $M_{t}(x, x)=x$, so by setting $x=y$ we have

$$
f(x) \geq \frac{1}{1-t} f(x)+\frac{1}{t} f(x)=\frac{1}{t(1-t)} f(x),
$$

which is equivalent to write $\left(t-t^{2}-1\right) f(x) \geq 0, \forall t \in(0,1)$. But since $f$ is non-negative we must have $t-t^{2}-1 \geq 0,0<t<1$ which is impossible and thus we got a contradiction. Hence, we must have $f(x) \leq 0$. 
In case when $f$ is nonnegative $\frac{1}{t}-\mathrm{M}_{\mathrm{t}} \mathrm{H}_{\mathrm{t}}-$ convex function, then

$$
f(M(t ; x, y)) \leq \frac{t(1-t) f(x) f(y)}{t f(x)+(1-t) f(y)},
$$

setting $x=y$ we have

$$
f(x) \leq t(1-t) f(x),
$$

and this is equivalent to write $(t(1-t)-1) f(x) \geq 0$, since $f$ is nonnegative we must have $t(1-t)-1 \geq 0$ which impossible for $t \in(0,1)$, which contradicts the nonnegativity assumption of $f$. Hence, $f \leq 0$.

Remark 1. There is no nonnegative $1-\mathrm{M}_{\mathrm{t}} \mathrm{A}_{\mathrm{t}}$-concave nor $1-\mathrm{M}_{\mathrm{t}} \mathrm{H}_{\mathrm{t}}$-convex functions, where $M_{t}=A_{t}, G_{t}, H_{t}$. The proof is simpler than that ones given above.

According to the previous discussion, we need to extend the classes $Q\left(I ; \mathrm{M}_{\mathrm{t}}, \mathrm{A}_{\mathrm{t}}\right), Q\left(I ; \mathrm{M}_{\mathrm{t}}, \mathrm{H}_{\mathrm{t}}\right)$, $P\left(I ; \mathrm{M}_{\mathrm{t}}, \mathrm{A}_{\mathrm{t}}\right)$, and $P\left(I ; \mathrm{M}_{\mathrm{t}}, \mathrm{H}_{\mathrm{t}}\right)$, by replacing the set of values of $f$ to be $\mathbb{R}$ instead of $(0, \infty)$. Consequently, we say that a function $f: I \rightarrow \mathbb{R}$

(1) is $\frac{1}{t}-\mathrm{M}_{\mathrm{t}} \mathrm{A}_{\mathrm{t}}$-concave, if $-f \in Q\left(I ; \mathrm{M}_{\mathrm{t}}, \mathrm{A}_{\mathrm{t}}\right)$, i.e.,

$$
f(\mathrm{M}(t ; x, y)) \geq \frac{1}{1-t} f(x)+\frac{1}{t} f(y),
$$

for all $x, y \in I$ and $t \in(0,1)$.

(2) is $\frac{1}{t}-\mathrm{M}_{\mathrm{t}} \mathrm{H}_{\mathrm{t}}$-convex, if $f \in Q\left(I ; \mathrm{M}_{\mathrm{t}}, \mathrm{H}_{\mathrm{t}}\right)$, i.e.,

$$
f(\mathrm{M}(t ; x, y)) \leq \frac{t(1-t) f(x) f(y)}{t f(x)+(1-t) f(y)},
$$

for all $x, y \in I$ and $t \in(0,1)$.

(3) is $1-\mathrm{M}_{\mathrm{t}} \mathrm{A}_{\mathrm{t}}$-concave, if $-f \in P\left(I ; \mathrm{M}_{\mathrm{t}}, \mathrm{A}_{\mathrm{t}}\right)$, i.e.,

$$
f(\mathrm{M}(t ; x, y)) \geq f(x)+f(y)
$$

for all $x, y \in I$ and $t \in(0,1)$.

(4) is $1-\mathrm{M}_{\mathrm{t}} \mathrm{H}_{\mathrm{t}}$-convex, if $f \in P\left(I ; \mathrm{M}_{\mathrm{t}}, \mathrm{H}_{\mathrm{t}}\right)$, i.e.,

$$
f(\mathrm{M}(t ; x, y)) \leq \frac{f(x) f(y)}{f(x)+f(y)},
$$

for all $x, y \in I$ and $t \in(0,1)$.

In the same way, there is no $\frac{1}{t}-\mathrm{M}_{\mathrm{t}} \mathrm{G}_{\mathrm{t}}$-concave function satisfies $f(x)>1$. To support this assertion, assume there exists $\frac{1}{t}-M_{t} G_{t}$-concave function, so that for Means $M_{t}$ and $G_{t}$, the reverse inequality of (1.3) holds for all $x, y \in I$ and $t \in(0,1)$.

$$
f(M(t ; x, y)) \geq[f(x)]^{\frac{1}{1-t}}[f(y)]^{\frac{1}{t}},
$$

since $M_{t}(x, x)=x$, by setting $x=y$ we get

$$
f(x) \geq[f(x)]^{\frac{1}{1-t}+\frac{1}{t}},
$$

since $f(x)>1$ and $t \in(0,1)$ then we must have $\frac{1}{1-t}+\frac{1}{t} \leq 1$ which is equivalent to write $1 \leq t(1-t)$ for all $t \in(0,1)$ and this is impossible, thus we have a contradiction. Hence, we must have $0 \leq f(x) \leq 1$. 
Remark 2. There is no $1-\mathrm{M}_{\mathrm{t}} \mathrm{G}_{\mathrm{t}}$-concave function satisfies $f(x)>1$. The proof is simpler than that ones given above.

As known, it is not easy to determine whether a given function is convex or not. Because of that, Jensen in [9] proved his famous characterization of convex functions. Simply, for a continuous functions $f$ defined on a real interval $I, f$ is convex if and only if

$$
f\left(\frac{x+y}{2}\right) \leq \frac{f(x)+f(y)}{2}
$$

for all $x, y \in I$.

In 1965, another characterization was presented by Popoviciu [16] (see also [13]), where he proved that the following theorem.

Theorem 1. Let $f: I \rightarrow \mathbb{R}$ be continuous. Then, $f$ is convex if and only if

$$
\frac{2}{3}\left[f\left(\frac{x+z}{2}\right)+f\left(\frac{y+z}{2}\right)+f\left(\frac{x+y}{2}\right)\right] \leq f\left(\frac{x+y+z}{3}\right)+\frac{f(x)+f(y)+f(z)}{3},
$$

for all $x, y, z \in I$, and the equality occurred by $f(x)=x, x \in I$.

The corresponding version of Popoviciu inequality for $\mathrm{G}_{\mathrm{t}} \mathrm{G}_{\mathrm{t}}$-convex (concave) function was presented by Niculescu [12], where he proved that for all $x, y, z \in I$ the inequality

$$
f^{2}(\sqrt{x z}) f^{2}(\sqrt{y z}) f^{2}(\sqrt{x y}) \leq(\geq) f^{3}(\sqrt[3]{x y z}) f(x) f(y) f(z)
$$

holds.

One of the most applicable benefits of Popoviciu's inequality is to maximize and/or minimize a given function (or certain real quantities) with out using derivatives, so that such type of inequalities plays an important role in Optimizations and Approximations. Another serious usefulness is to generalize some old famous inequalities, e.g., the Popoviciu's inequality can be considered as an elegant generalization of Hlawka's inequality using convexity as a simple tool of geometry. For any real numbers $x, y, z$, the Hlawka's inequality reads:

$$
|x|+|y|+|z|+|x+y+z| \geq|x+z|+|z+y|+|x+y| \text {. }
$$

D. Smiley \& M. Smiley [20] (see also [17], p. 756), interpreted Hlawka's inequality geometrically by saying that: "the total length over all sums of pairs from three vectors is not greater than the perimeter of the quadrilateral defined by the three vectors." For comprehensive history regarding Hlawka's inequality see the recent work of Fechner [6] and the classical monograph of Mitrinović et al. [11].

One may count third benefit, the extended version of Popoviciu's inequality to several variables was not possible without the help of Hlawka's inequality, as it inspired the authors of [3] to develop a higher dimensional analogue of Popoviciu's inequality based on his characterization. Interesting generalizations and counterparts of Popoviciu inequality with some ramified consequences can be found in [14] and [19].

So that, as Popoviciu's inequality one of the most popular generalization of Hlawka's inequality, and due to its important usefulness, in this work we establish some Popoviciu type inequalities with respect to different types of means. More preciously, for $h$-AN-convex functions several inequalities of Popoviciu type are proved. In this way, we extend Hlawka's inequality based on the geometric structure used under an $h$-AN-convex mappings. 


\section{Popoviciu type inequalities for $h-A_{t} N_{t}$-COnvex functions}

In this section, we prove the corresponding version of Popoviciu inequality for the classes $h$ - $\mathrm{A}_{\mathrm{t}} \mathrm{N}_{\mathrm{t}}$-convex functions, where $\mathrm{N}_{\mathrm{t}}=\mathrm{A}_{\mathrm{t}}, \mathrm{G}_{\mathrm{t}}, \mathrm{H}_{\mathrm{t}}$.

First of all, we recall that, a function $h: I \rightarrow \mathbb{R}$ is said to be

(1) additive if $h(s+t)=h(s)+h(t)$,

(2) subadditive if $h(s+t) \leq h(s)+h(t)$,

(3) superadditive if $h(s+t) \geq h(s)+h(t)$,

for all $s, t \in I$. For instance, let $h: I \rightarrow(0, \infty)$ given by $h(t)=t^{k}, t>0$. Then $h$ is

(1) additive if $k=1$.

(2) subadditive if $k \in(-\infty,-1] \cup[0,1)$.

(3) superadditive if $k \in(-1,0) \cup(1, \infty)$.

We note here, in all next results and for the classes $\frac{1}{t}-\mathrm{M}_{\mathrm{t}} \mathrm{A}_{\mathrm{t}}$-concave, $\frac{1}{t}-\mathrm{M}_{\mathrm{t}} \mathrm{H}_{\mathrm{t}}$-convex , 1$\mathrm{M}_{\mathrm{t}} \mathrm{A}_{\mathrm{t}}$-concave, and 1- $\mathrm{M}_{\mathrm{t}} \mathrm{H}_{\mathrm{t}}$-convex functions, $f$ can be defined to be $f: I \rightarrow \mathbb{R}, I \subseteq(0, \infty)$.

2.1. The case when $f$ is $h$ - $A_{t} A_{t}$-convex. Now, we are ready to state our first main result.

Theorem 2. Let $h: I \rightarrow(0, \infty)$ be a non-negative super(sub)additive function. If $f: I \rightarrow$ $(0, \infty)$ be an $h-\mathrm{A}_{\mathrm{t}} \mathrm{A}_{\mathrm{t}}$-convex (-concave) function, then

$$
\begin{aligned}
f\left(\frac{x+z}{2}\right)+f\left(\frac{y+z}{2}\right) & +f\left(\frac{x+y}{2}\right) \\
\leq & (\geq) h(3 / 2) f\left(\frac{x+y+z}{3}\right)+h(1 / 2)[f(x)+f(y)+f(z)]
\end{aligned}
$$

for all $x, y, z \in I$.

Proof. $f$ is $h-\mathrm{A}_{\mathrm{t}} \mathrm{A}_{\mathrm{t}}$-convex iff the inequality

$$
f(t \alpha+(1-t) \beta) \leq h(t) f(\alpha)+h(1-t) f(\beta), \quad 0 \leq t \leq 1,
$$

holds for all $\alpha, \beta \in I$. Assume that $x \leq y \leq z$. If $y \leq \frac{x+y+z}{3}$, then

$$
\frac{x+y+z}{3} \leq \frac{x+z}{2} \leq z \text { and } \frac{x+y+z}{3} \leq \frac{y+z}{2} \leq z,
$$

so that there exist two numbers $s, t \in[0,1]$ satisfying

$$
\frac{x+z}{2}=s\left(\frac{x+y+z}{3}\right)+(1-s) z,
$$

and

$$
\frac{y+z}{2}=t\left(\frac{x+y+z}{3}\right)+(1-t) z .
$$

Summing up, we get $(x+y-2 z)\left(s+t-\frac{3}{2}\right)=0$. If $x+y-2 z=0$, then $x=y=z$, and Popoviciu's inequality holds.

If $s+t=\frac{3}{2}$, then since $f$ is $\mathrm{A}_{\mathrm{t}} \mathrm{A}_{\mathrm{t}}$-convex, we have

$$
\begin{aligned}
& f\left(\frac{x+z}{2}\right)=f\left[s\left(\frac{x+y+z}{3}\right)+(1-s) z\right] \leq h(s) f\left(\frac{x+y+z}{3}\right)+h(1-s) f(z), \\
& f\left(\frac{y+z}{2}\right)=f\left[t\left(\frac{x+y+z}{3}\right)+(1-t) z\right] \leq h(t) f\left(\frac{x+y+z}{3}\right)+h(1-t) f(z),
\end{aligned}
$$


and

$$
f\left(\frac{x+y}{2}\right) \leq h(1 / 2)[f(x)+f(y)] .
$$

Summing up these inequalities taking into account that $h$ is superadditive we get

$$
\begin{aligned}
f\left(\frac{x+z}{2}\right)+f\left(\frac{y+z}{2}\right)+f\left(\frac{x+y}{2}\right) \\
\leq h(s) f\left(\frac{x+y+z}{3}\right)+h(1-s) f(z)+h(t) f\left(\frac{x+y+z}{3}\right)+h(1-t) f(z) \\
\quad \quad+h(1 / 2)[f(x)+f(y)] \\
=[h(s)+h(t)] f\left(\frac{x+y+z}{3}\right)+[h(1-s)+h(1-t)] f(z)+h(1 / 2)[f(x)+f(y)] \\
\leq h(s+t) f\left(\frac{x+y+z}{3}\right)+h(2-s-t) f(z)+h(1 / 2)[f(x)+f(y)] \\
=h(3 / 2) f\left(\frac{x+y+z}{3}\right)+h(1 / 2) f(z)+h(1 / 2)[f(x)+f(y)] \\
=h(3 / 2) f\left(\frac{x+y+z}{3}\right)+h(1 / 2)[f(x)+f(y)+f(z)],
\end{aligned}
$$

as desired in (2.1).

Remark 3. In (2.1), setting $z=y$, then we have

$$
2 f\left(\frac{x+y}{2}\right)+f(y) \leq(\geq) h(3 / 2) f\left(\frac{x+2 y}{3}\right)+h(1 / 2)[f(x)+2 f(y)] .
$$

for all $x, y \in I$.

Remark 4. In (2.1), setting $z=y$ then we get

$$
2 f\left(\frac{x+y}{2}\right)+f(y) \leq(\geq) h(3 / 2) f\left(\frac{x+2 y}{3}\right)+h(1 / 2)[f(x)+2 f(y)],
$$

for all $x, y \in I$.

Corollary 1. Let $h: I \rightarrow(0, \infty)$ be a non-negative super(sub)additive function. If $f: I \rightarrow$ $(0, \infty)$ be an $\mathrm{A}_{\mathrm{t}} \mathrm{A}_{\mathrm{t}}$-convex (concave) function, then

$$
\frac{2}{3}\left[f\left(\frac{x+z}{2}\right)+f\left(\frac{y+z}{2}\right)+f\left(\frac{x+y}{2}\right)\right] \leq(\geq) f\left(\frac{x+y+z}{3}\right)+\frac{f(x)+f(y)+f(z)}{3},
$$

for all $x, y, z \in I$. The equality holds when $f$ is affine.

Example 1. (1) Let $f(x)=x^{p}, p \geq 1$ then $f$ is $\mathrm{A}_{\mathrm{t}} \mathrm{A}_{\mathrm{t}}$-convex for all $x>0$. Applying Corollary 1, we get

$$
\frac{2}{3}\left[\left(\frac{x+z}{2}\right)^{p}+\left(\frac{y+z}{2}\right)^{p}+\left(\frac{x+y}{2}\right)^{p}\right] \leq\left(\frac{x+y+z}{3}\right)^{p}+\frac{x^{p}+y^{p}+z^{p}}{3},
$$

for all $x, y, z>0$.

(2) Let $f(x)=-\log x$, then $f$ is $\mathrm{A}_{\mathrm{t}} \mathrm{A}_{\mathrm{t}}$-convex for all $0<x<1$. Applying Corollary 1, we get

$$
(x+z)^{2}(y+z)^{2}(x+y)^{2} \geq \frac{64}{27}(x+y+z)^{3}(x y z),
$$

for all $1>x, y, z>0$. 
Corollary 2. If $f: I \rightarrow \mathbb{R}$ be an $\frac{1}{t}-\mathrm{A}_{\mathrm{t}} \mathrm{A}_{\mathrm{t}}$-concave function, then

$$
\begin{aligned}
& \frac{3}{2}\left[f\left(\frac{x+z}{2}\right)+f\left(\frac{y+z}{2}\right)+f\left(\frac{x+y}{2}\right)\right] \\
& \leq(\geq) f\left(\frac{x+y+z}{3}\right)+3[f(x)+f(y)+f(z)],
\end{aligned}
$$

for all $x, y, z \in I$.

Example 2. Let $f(x)=\log x$, then $f$ is an $\frac{1}{t}-\mathrm{A}_{\mathrm{t}} \mathrm{A}_{\mathrm{t}}$-concave for $0<x<1$. Applying Corollary 2, we get

$$
(x+z)^{3}(y+z)^{3}(x+y)^{3} \geq \frac{512}{9}(x+y+z)^{2}(x y z)^{6},
$$

for all $0<x, y, z<1$.

Corollary 3. If $f: I \rightarrow \mathbb{R}$ be an $1-\mathrm{A}_{\mathrm{t}} \mathrm{A}_{\mathrm{t}}$-concave function, then

$$
f\left(\frac{x+z}{2}\right)+f\left(\frac{y+z}{2}\right)+f\left(\frac{x+y}{2}\right) \leq(\geq) f\left(\frac{x+y+z}{3}\right)+f(x)+f(y)+f(z),
$$

for all $x, y, z \in I$.

Example 3. Let $f(x)=\log x$, which is a non-negative $1-\mathrm{A}_{\mathrm{t}} \mathrm{A}_{\mathrm{t}}$-concave for all $0<x<1$. Applying Corollary 3, we get

$$
(x+z)(y+z)(x+y) \geq \frac{8}{3}(x+y+z)(x y z),
$$

for all $0<x, y, z<1$.

Corollary 4. In Theorem 2.

(1) If $h: J \rightarrow(0, \infty)$ is a nonnegative is superadditive and $f: I \rightarrow(0, \infty)$ is an $h-\mathrm{A}_{\mathrm{t}} \mathrm{A}_{\mathrm{t}}$ convex and subadditive, then

$$
\begin{aligned}
f(x+y+z) \leq & f\left(\frac{x+z}{2}\right)+f\left(\frac{y+z}{2}\right)+f\left(\frac{x+y}{2}\right) \\
\leq & h(3 / 2) f\left(\frac{x+y+z}{3}\right)+h(1 / 2)[f(x)+f(y)+f(z)] \\
& \leq h(3 / 2)\left[f\left(\frac{x}{3}\right)+f\left(\frac{y}{3}\right)+f\left(\frac{z}{3}\right)\right]+h(1 / 2)[f(x)+f(y)+f(z)],
\end{aligned}
$$

for all $x, y, z \in I$. If $h$ is nonnegative subadditive on $J$ and $f$ is an $h-\mathrm{A}_{\mathrm{t}} \mathrm{A}_{\mathrm{t}}$-concave and superadditive, then the inequality is reversed.

(2) If $h: J \rightarrow(0, \infty)$ is a nonnegative is superadditive and $f: I \rightarrow(0, \infty)$ is an $h-\mathrm{A}_{\mathrm{t}} \mathrm{A}_{\mathrm{t}}$ convex and superadditive, then

$$
\begin{aligned}
f\left(\frac{x+z}{2}\right)+f\left(\frac{y+z}{2}\right)+f\left(\frac{x+y}{2}\right) & \\
\leq h(3 / 2) f\left(\frac{x+y+z}{3}\right) & +h(1 / 2)[f(x)+f(y)+f(z)] \\
\leq & h(3 / 2) f\left(\frac{x+y+z}{3}\right)+h(1 / 2) f(x+y+z),
\end{aligned}
$$

for all $x, y, z \in I$. If $h$ is a nonnegative is subadditive and $f$ is an $h-\mathrm{A}_{\mathrm{t}} \mathrm{A}_{\mathrm{t}}$-concave and subadditive, then the inequality is reversed. 


\subsection{The case when $f$ is $h-A_{t} G_{t}$-convex.}

Theorem 3. Let $h: I \rightarrow(0, \infty)$ be a non-negative super(sub)additive function. If $f: I \rightarrow$ $(0, \infty)$ be an $h-A_{t} G_{t}$-convex (-concave) function, then

$$
\begin{aligned}
& f\left(\frac{x+z}{2}\right) f\left(\frac{y+z}{2}\right) f\left(\frac{x+y}{2}\right) \\
& \leq(\geq)\left[f\left(\frac{x+y+z}{3}\right)\right]^{h(3 / 2)}[f(x) f(y) f(z)]^{h(1 / 2)},
\end{aligned}
$$

for all $x, y, z \in I$.

Proof. $f$ is $h-\mathrm{A}_{\mathrm{t}} \mathrm{G}_{\mathrm{t}}$-convex iff the inequality

$$
f(t \alpha+(1-t) \beta) \leq[f(\alpha)]^{h(t)}[f(\beta)]^{h(1-t)}, \quad 0 \leq t \leq 1
$$

holds for all $\alpha, \beta \in I$. As in the proof of Theorem 2, we have $(x+y-2 z)\left(s+t-\frac{3}{2}\right)=0$. If $x+y-2 z=0$, then $x=y=z$, and Popoviciu's inequality holds.

If $s+t=\frac{3}{2}$, then since $f$ is $\mathrm{A}_{\mathrm{t}} \mathrm{G}_{\mathrm{t}}$-convex, we have

$$
\begin{aligned}
& f\left(\frac{x+z}{2}\right)=f\left[s\left(\frac{x+y+z}{3}\right)+(1-s) z\right] \leq\left[f\left(\frac{x+y+z}{3}\right)\right]^{h(s)}[f(z)]^{h(1-s)} \\
& f\left(\frac{y+z}{2}\right)=f\left[t\left(\frac{x+y+z}{3}\right)+(1-t) z\right] \leq\left[f\left(\frac{x+y+z}{3}\right)\right]^{h(t)}[f(z)]^{h(1-t)}
\end{aligned}
$$

and

$$
f\left(\frac{x+y}{2}\right) \leq[f(x) f(y)]^{h(1 / 2)}
$$

Multiplying these inequalities we get

$$
\begin{aligned}
& f\left(\frac{x+z}{2}\right) f\left(\frac{y+z}{2}\right) f\left(\frac{x+y}{2}\right) \\
& \leq\left[f\left(\frac{x+y+z}{3}\right)\right]^{h(s)}[f(z)]^{h(1-s)}\left[f\left(\frac{x+y+z}{3}\right)\right]^{h(t)}[f(z)]^{h(1-t)}[f(x) f(y)]^{h(1 / 2)} \\
& =\left[f\left(\frac{x+y+z}{3}\right)\right]^{h(s)+h(t)}[f(z)]^{h(1-s)+h(1-t)}[f(x) f(y)]^{h(1 / 2)} \\
& \leq\left[f\left(\frac{x+y+z}{3}\right)\right]^{h(s+t)}[f(z)]^{h(2-s-t)}[f(x) f(y)]^{\frac{1}{2}} \\
& =\left[f\left(\frac{x+y+z}{3}\right)\right]^{h(3 / 2)}[f(x) f(y) f(z)]^{h(1 / 2)},
\end{aligned}
$$

which proves the inequality in (2.2).

Remark 5. In (2.2), setting $z=y$ then we have

$$
f^{2}\left(\frac{x+y}{2}\right) f(y) \leq(\geq)\left[f\left(\frac{x+2 y}{3}\right)\right]^{h(3 / 2)}\left[f(x) f^{2}(y)\right]^{h(1 / 2)}
$$

for all $x, y \in I$. 
Corollary 5. If $f: I \rightarrow(0, \infty)$ be an $\mathrm{A}_{\mathrm{t}} \mathrm{G}_{\mathrm{t}}$-convex function, then

$$
f^{2}\left(\frac{x+z}{2}\right) f^{2}\left(\frac{y+z}{2}\right) f^{2}\left(\frac{x+y}{2}\right) \leq f^{3}\left(\frac{x+y+z}{3}\right) f(x) f(y) f(z),
$$

for all $x, y, z \in I$. The equality occurred for $f(x)=\mathrm{e}^{x}, x>0$.

Example 4. $f(x)=\cosh (x), x \in \mathbb{R}$ is $\mathrm{A}_{\mathrm{t}} \mathrm{G}_{\mathrm{t}}$-convex function. Applying Corollary 5 we get

$$
\begin{aligned}
\cosh ^{2}\left(\frac{x+z}{2}\right) \cosh ^{2}\left(\frac{y+z}{2}\right) \cosh ^{2}\left(\frac{x+y}{2}\right) & \\
\leq & \cosh ^{3}\left(\frac{x+y+z}{3}\right) \cosh (x) \cosh (y) \cosh (z)
\end{aligned}
$$

Corollary 6. If $f: I \rightarrow(0, \infty)$ be an $\frac{1}{t}-\mathrm{A}_{\mathrm{t}} \mathrm{G}_{\mathrm{t}}$-concave function, then

$$
f^{3}\left(\frac{x+z}{2}\right) f^{3}\left(\frac{y+z}{2}\right) f^{3}\left(\frac{x+y}{2}\right) \geq f^{2}\left(\frac{x+y+z}{3}\right) f^{6}(x) f^{6}(y) f^{6}(z),
$$

for all $x, y, z \in I$.

Example 5. $f(x)=\arcsin (x)$, is $\frac{1}{t}-\mathrm{A}_{\mathrm{t}} \mathrm{G}_{\mathrm{t}}$-concave for $x \in[0,1]$. Applying Corollary 6 we get

$$
\begin{aligned}
\arcsin ^{3}\left(\frac{x+z}{2}\right) \arcsin ^{3}\left(\frac{y+z}{2}\right) & \arcsin ^{3}\left(\frac{x+y}{2}\right) \\
& \geq \arcsin ^{2}\left(\frac{x+y+z}{3}\right) \arcsin ^{6}(x) \arcsin ^{6}(y) \arcsin ^{6}(z),
\end{aligned}
$$

for all $0 \leq x, y, z \leq 1$.

Corollary 7. If $f: I \rightarrow(0, \infty)$ be an $1-\mathrm{A}_{\mathrm{t}} \mathrm{G}_{\mathrm{t}}$-concave function, then

$$
f\left(\frac{x+z}{2}\right) f\left(\frac{y+z}{2}\right) f\left(\frac{x+y}{2}\right) \leq(\geq) f\left(\frac{x+y+z}{3}\right) f(x) f(y) f(z),
$$

for all $x, y, z \in I$.

Example 6. Let $f(x)=\arcsin (x)$, is $1-\mathrm{A}_{\mathrm{t}} \mathrm{G}_{\mathrm{t}}$-concave for $x \in[0,1]$. Applying Corollary 7 we get

$$
\begin{aligned}
\arcsin \left(\frac{x+z}{2}\right) \arcsin \left(\frac{y+z}{2}\right) \arcsin & \left(\frac{x+y}{2}\right) \\
& \geq \arcsin \left(\frac{x+y+z}{3}\right) \arcsin (x) \arcsin (y) \arcsin (z),
\end{aligned}
$$

for all $0 \leq x, y, z \leq 1$.

Corollary 8. In Theorem 3.

(1) If $f: I \rightarrow(0, \infty)$ is an $h-\mathrm{A}_{\mathrm{t}} \mathrm{G}_{\mathrm{t}}$-convex and submultiplicative,

$$
\begin{aligned}
f\left(\frac{(x+z)(y+z)(x+y)}{8}\right) \leq f\left(\frac{x+z}{2}\right) & f\left(\frac{y+z}{2}\right) f\left(\frac{x+y}{2}\right) \\
\leq & {\left[f\left(\frac{x+y+z}{3}\right)\right]^{h(3 / 2)}[f(x) f(y) f(z)]^{h(1 / 2)}, }
\end{aligned}
$$

for all $x, y, z \in I$. If $f$ is an $h-\mathrm{A}_{\mathrm{t}} \mathrm{G}_{\mathrm{t}}$-concave and supermultiplicative, then the inequality is reversed. 
(2) If $f: I \rightarrow(0, \infty)$ is an $h-\mathrm{A}_{\mathrm{t}} \mathrm{G}_{\mathrm{t}}$-convex and supermultiplicative, then

$$
\begin{aligned}
f\left(\frac{x+z}{2}\right) f\left(\frac{y+z}{2}\right) & f\left(\frac{x+y}{2}\right) \\
\leq\left[f\left(\frac{x+y+z}{3}\right)\right]^{h(3 / 2)}[f(x) f(y) f(z)]^{h(1 / 2)} & \leq\left[f\left(\frac{x+y+z}{3}\right)\right]^{h(3 / 2)}[f(x y z)]^{h(1 / 2)},
\end{aligned}
$$

for all $x, y, z \in I$. If $f$ is an $h-\mathrm{A}_{\mathrm{t}} \mathrm{A}_{\mathrm{t}}$-concave and submultiplicative, then the inequality is reversed.

Corollary 9. In Theorem 3.

(1) If $f: I \rightarrow(0, \infty)$ is an $h-\mathrm{A}_{\mathrm{t}} \mathrm{G}_{\mathrm{t}}$-convex and superadditive,

$$
\begin{aligned}
{\left[f\left(\frac{x}{2}\right)+f\left(\frac{z}{2}\right)\right]\left[f\left(\frac{y}{2}\right)+\right.} & \left.f\left(\frac{z}{2}\right)\right]\left[f\left(\frac{x}{2}\right)+f\left(\frac{y}{2}\right)\right] \\
\leq f\left(\frac{x+z}{2}\right) & f\left(\frac{y+z}{2}\right) f\left(\frac{x+y}{2}\right) \\
& \leq\left[f\left(\frac{x+y+z}{3}\right)\right]^{h(3 / 2)}[f(x) f(y) f(z)]^{h(1 / 2)}
\end{aligned}
$$

for all $x, y, z \in I$. If $f$ is an $h-\mathrm{A}_{\mathrm{t}} \mathrm{G}_{\mathrm{t}}$-concave and subadditive, then the inequality is reversed.

(2) If $f: I \rightarrow(0, \infty)$ is an $h-\mathrm{A}_{\mathrm{t}} \mathrm{G}_{\mathrm{t}}$-convex and subadditive, then

$$
\begin{aligned}
f\left(\frac{x+z}{2}\right) f\left(\frac{y+z}{2}\right) & f\left(\frac{x+y}{2}\right) \\
\leq & {\left[f\left(\frac{x+y+z}{3}\right)\right]^{h(3 / 2)}[f(x) f(y) f(z)]^{h(1 / 2)} } \\
\leq & {\left[f\left(\frac{x}{3}\right)+f\left(\frac{y}{3}\right)+f\left(\frac{z}{3}\right)\right]^{h(3 / 2)}[f(x) f(y) f(z)]^{h(1 / 2)}, }
\end{aligned}
$$

for all $x, y, z \in I$. If $f$ is an $h-\mathrm{A}_{\mathrm{t}} \mathrm{G}_{\mathrm{t}}$-concave and submultiplicative, then the inequality is reversed.

\subsection{The case when $f$ is $h-\mathrm{A}_{\mathrm{t}} \mathrm{H}_{\mathrm{t}}$-convex.}

Theorem 4. Let $h: I \rightarrow(0, \infty)$ be a non-negative super(sub)additive function. If $f: I \rightarrow$ $(0, \infty)$ is an $h-\mathrm{A}_{\mathrm{t}} \mathrm{H}_{\mathrm{t}}$-concave (-convex), then

$$
\begin{aligned}
& \frac{1}{f\left(\frac{x+z}{2}\right)}+\frac{1}{f\left(\frac{y+z}{2}\right)}+\frac{1}{f\left(\frac{x+y}{2}\right)} \\
& \leq(\geq) h(1 / 2)\left[\frac{1}{f(y)}+\frac{1}{f(x)}+\frac{1}{f(z)}\right]+\frac{h(3 / 2)}{f\left(\frac{x+y+z}{3}\right)},
\end{aligned}
$$

for all $x, y, z \in I$.

Proof. $f$ is $h-\mathrm{A}_{\mathrm{t}} \mathrm{H}_{\mathrm{t}}$-convex iff the inequality

$$
f(t \alpha+(1-t) \beta) \leq \frac{f(\alpha) f(\beta)}{h(1-t) f(\alpha)+h(t) f(\beta)}, \quad 0 \leq t \leq 1
$$


holds for all $\alpha, \beta \in I$. As in the proof of Theorem 2, we have $(x+y-2 z)\left(s+t-\frac{3}{2}\right)=0$. If $x+y-2 z=0$, then $x=y=z$, and Popoviciu's inequality holds.

If $s+t=\frac{3}{2}$, then since $f$ is $\mathrm{A}_{\mathrm{t}} \mathrm{H}_{\mathrm{t}}$-concave, we have

$$
f\left(\frac{x+z}{2}\right)=f\left[s\left(\frac{x+y+z}{3}\right)+(1-s) z\right] \geq \frac{f\left(\frac{x+y+z}{3}\right) f(z)}{h(1-s) f\left(\frac{x+y+z}{3}\right)+h(s) f(z)},
$$

and this equivalent to write

$$
\frac{1}{f\left(\frac{x+z}{2}\right)} \leq \frac{h(1-s) f\left(\frac{x+y+z}{3}\right)+h(s) f(z)}{f\left(\frac{x+y+z}{3}\right) f(z)}
$$

similarly,

$$
f\left(\frac{y+z}{2}\right)=f\left[t\left(\frac{x+y+z}{3}\right)+(1-t) z\right] \geq \frac{f\left(\frac{x+y+z}{3}\right) f(z)}{h(1-t) f\left(\frac{x+y+z}{3}\right)+h(t) f(z)},
$$

which equivalent to write

$$
\frac{1}{f\left(\frac{y+z}{2}\right)} \leq \frac{h(1-t) f\left(\frac{x+y+z}{3}\right)+h(t) f(z)}{f\left(\frac{x+y+z}{3}\right) f(z)},
$$

and

$$
\begin{aligned}
f\left(\frac{x+y}{2}\right) & \geq \frac{f(x) f(y)}{h(1 / 2)(f(x)+f(y))} \\
\Longleftrightarrow & \frac{1}{f\left(\frac{x+y}{2}\right)} \leq \frac{h(1 / 2)(f(x)+f(y))}{f(x) f(y)},
\end{aligned}
$$

Summing the inequalities (2.4)-(2.6), we get

$$
\begin{aligned}
& \quad \frac{1}{f\left(\frac{x+z}{2}\right)}+\frac{1}{f\left(\frac{y+z}{2}\right)}+\frac{1}{f\left(\frac{x+y}{2}\right)} \\
& \leq \frac{h(1-s) f\left(\frac{x+y+z}{3}\right)+h(s) f(z)}{f\left(\frac{x+y+z}{3}\right) f(z)}+\frac{h(1-t) f\left(\frac{x+y+z}{3}\right)+h(t) f(z)}{f\left(\frac{x+y+z}{3}\right) f(z)} \\
& \quad \quad+\frac{h(1 / 2)(f(x)+f(y))}{f(x) f(y)} \\
& =\frac{[h(1-s)+h(1-t)] f\left(\frac{x+y+z}{3}\right)+[h(s)+h(t)] f(z)}{f\left(\frac{x+y+z}{3}\right) f(z)} \\
& \quad+\frac{h(1 / 2)(f(x)+f(y))}{f(x) f(y)} \\
& \leq \frac{h(2-s-t) f\left(\frac{x+y+z}{3}\right)+h(s+t) f(z)}{f\left(\frac{x+y+z}{3}\right) f(z)}+\frac{h(1 / 2)(f(x)+f(y))}{f(x) f(y)} \\
& =\frac{h(1 / 2) f\left(\frac{x+y+z}{3}\right)+h(3 / 2) f(z)}{f\left(\frac{x+y+z}{3}\right) f(z)}+\frac{h(1 / 2)(f(x)+f(y))}{f(x) f(y)} \\
& =h(1 / 2)\left[\frac{1}{f(y)}+\frac{1}{f(x)}+\frac{1}{f(z)}\right]+\frac{h(3 / 2)}{f\left(\frac{x+y+z}{3}\right)},
\end{aligned}
$$

which proves the inequality in (2.3). 
Remark 6. In (2.3), setting $z=y$, then we have

$$
\frac{2}{f\left(\frac{x+y}{2}\right)}+\frac{1}{f\left(\frac{y+z}{2}\right)} \leq(\geq) h(1 / 2)\left[\frac{2}{f(y)}+\frac{1}{f(x)}\right]+\frac{h(3 / 2)}{f\left(\frac{x+2 y}{3}\right)},
$$

for all $x, y \in I$.

Corollary 10. If $f: I \rightarrow(0, \infty)$ is an $\mathrm{A}_{\mathrm{t}} \mathrm{H}_{\mathrm{t}}$-concave (convex), then

$$
\frac{2}{3}\left[\frac{1}{f\left(\frac{x+z}{2}\right)}+\frac{1}{f\left(\frac{y+z}{2}\right)}+\frac{1}{f\left(\frac{x+y}{2}\right)}\right] \leq(\geq) \frac{1}{3}\left[\frac{1}{f(y)}+\frac{1}{f(x)}+\frac{1}{f(z)}\right]+\frac{1}{f\left(\frac{x+y+z}{3}\right)},
$$

for all $x, y, z \in I$. The equality holds with $f(x)=\frac{1}{x}, x>0$.

Example 7. Let $f(x)=x^{p}, p \geq 1$. Then $\mathrm{A}_{\mathrm{t}} \mathrm{H}_{\mathrm{t}}$-concave for $x \geq 1$. Applying Corollary 10, we get

$$
\frac{2}{3}\left[\left(\frac{x+z}{2}\right)^{-p}+\left(\frac{y+z}{2}\right)^{-p}+\left(\frac{x+y}{2}\right)^{-p}\right] \leq \frac{x^{-p}+y^{-p}+z^{-p}}{3}+\left(\frac{x+y+z}{3}\right)^{-p}
$$

for all $x, y, z \geq 1$.

Corollary 11. If $f: I \rightarrow(0, \infty)$ is an $\frac{1}{t}-\mathrm{A}_{\mathrm{t}} \mathrm{H}_{\mathrm{t}}$-convex, then

$$
\frac{3}{2}\left[\frac{1}{f\left(\frac{x+z}{2}\right)}+\frac{1}{f\left(\frac{y+z}{2}\right)}+\frac{1}{f\left(\frac{x+y}{2}\right)}\right] \leq 3\left[\frac{1}{f(y)}+\frac{1}{f(x)}+\frac{1}{f(z)}\right]+\frac{1}{f\left(\frac{x+y+z}{3}\right)},
$$

for all $x, y, z \in I$.

Example 8. Let $f(x)=-\log (x), x \supsetneqq 1$. Then, $f$ is $\frac{1}{t}-\mathrm{A}_{\mathrm{t}} \mathrm{H}_{\mathrm{t}}$-convex for $x \supsetneqq 1$. Applying Corollary 11, we get

$$
\frac{3}{2}\left[\frac{1}{\log \left(\frac{x+z}{2}\right)}+\frac{1}{\log \left(\frac{y+z}{2}\right)}+\frac{1}{\log \left(\frac{x+y}{2}\right)}\right] \leq 3\left(\frac{1}{\log x}+\frac{1}{\log y}+\frac{1}{\log z}\right)+\log (x y z)^{\frac{1}{3}},
$$

for all $x, y, z \supsetneqq 1$.

Corollary 12. If $f: I \rightarrow(0, \infty)$ is an $1-\mathrm{A}_{\mathrm{t}} \mathrm{H}_{\mathrm{t}}$-convex, then

$$
\frac{1}{f\left(\frac{x+z}{2}\right)}+\frac{1}{f\left(\frac{y+z}{2}\right)}+\frac{1}{f\left(\frac{x+y}{2}\right)} \leq\left[\frac{1}{f(y)}+\frac{1}{f(x)}+\frac{1}{f(z)}\right]+\frac{1}{f\left(\frac{x+y+z}{3}\right)},
$$

for all $x, y, z \in I$.

Example 9. Let $f(x)=-\log (x), x \supsetneqq 1$. Then, $f$ is $1-\mathrm{A}_{\mathrm{t}} \mathrm{H}_{\mathrm{t}}$-convex on $x \supsetneqq 1$. Applying Corollary 11, we get

$$
\frac{1}{\log \left(\frac{x+z}{2}\right)}+\frac{1}{\log \left(\frac{y+z}{2}\right)}+\frac{1}{\log \left(\frac{x+y}{2}\right)} \leq \frac{1}{\log x}+\frac{1}{\log y}+\frac{1}{\log z}+\log (x y z)^{\frac{1}{3}},
$$

for all $x, y, z \supsetneqq 1$.

\section{Popoviciu inequalities for $h$ - $\mathrm{G}_{\mathrm{t}} \mathrm{N}_{\mathrm{t}}$-COnvex functions}

In this section, we prove the corresponding version of Popoviciu inequality for the classes $h$ - $\mathrm{G}_{\mathrm{t}} \mathrm{N}_{\mathrm{t}}$-convex functions, where $\mathrm{N}_{\mathrm{t}}=\mathrm{A}_{\mathrm{t}}, \mathrm{G}_{\mathrm{t}}, \mathrm{H}_{\mathrm{t}}$. 


\subsection{The case when $f$ is $h$ - $G_{t} A_{t}$-convex.}

Theorem 5. Let $h: I \rightarrow(0, \infty)$ be a non-negative super(sub)additive function. If $f: I \rightarrow$ $(0, \infty)$ is $h-\mathrm{G}_{\mathrm{t}} \mathrm{A}_{\mathrm{t}}$-convex (-concave) function, then

$$
\begin{array}{r}
f(\sqrt{x z})+f(\sqrt{y z})+f(\sqrt{x y}) \\
\leq(\geq) h(3 / 2) f(\sqrt[3]{x y z})+h(1 / 2)[f(x)+f(y)+f(z)],
\end{array}
$$

for all $x, y, z \in I$.

Proof. $f$ is $h$ - $\mathrm{G}_{\mathrm{t}} \mathrm{A}_{\mathrm{t}}$-convex iff the inequality

$$
f\left(\alpha^{t} \beta^{1-t}\right) \leq h(t) f(\alpha)+h(1-t) f(\beta), \quad 0 \leq t \leq 1
$$

holds for all $\alpha, \beta \in I$. Assume that $x \leq y \leq z$. If $y \leq(x y z)^{1 / 3}$, then

$$
(x y z)^{1 / 3} \leq(x z)^{1 / 2} \leq z \text { and }(x y z)^{1 / 3} \leq(y z)^{1 / 2} \leq z,
$$

so that there exist two numbers $s, t \in[0,1]$ satisfying

$$
(x z)^{1 / 2}=(x y z)^{s / 3} z^{1-s}
$$

and

$$
(y z)^{1 / 2}=(x y z)^{t / 3} z^{1-t}
$$

Multiplying the above equations, we get

$$
(x y z)^{1 / 2} z^{1 / 2}=(x y z)^{(s+t) / 3} z^{2-(s+t)}
$$

or

$$
(x y z)^{\frac{(s+t)}{3}-\frac{1}{2}} z^{2-(s+t)-\frac{1}{2}}=1 .
$$

If $x y z^{2}=1$, then $x=y=z$, and Popoviciu's inequality holds.

If $s+t=\frac{3}{2}$, then since $f$ is $\mathrm{G}_{\mathrm{t}} \mathrm{A}_{\mathrm{t}}$-convex, we have

$$
\begin{aligned}
& f(\sqrt{x z})=f\left[(x y z)^{s / 3} z^{1-s}\right] \leq h(s)[f(\sqrt[3]{x y z})]+h(1-s)[f(z)] \\
& f(\sqrt{y z})=f\left[(x y z)^{t / 3} z^{1-t}\right] \leq h(t)[f(\sqrt[3]{x y z})]+h(1-t)[f(z)] \\
& f(\sqrt{x y}) \leq h\left(\frac{1}{2}\right)[f(x)+f(y)]
\end{aligned}
$$

Summing up these inequalities, we get

$$
\begin{aligned}
f & (\sqrt{x z})+f(\sqrt{y z})+f(\sqrt{x y}) \\
\leq & h(s) f(\sqrt[3]{x y z})+h(1-s) f(z)+h(t) f(\sqrt[3]{x y z})+h(1-t) f(z) \\
& \quad+h(1 / 2)[f(x)+f(y)] \\
= & {[h(s)+h(t)] f(\sqrt[3]{x y z})+[h(1-s)+h(1-t)] f(z)+h(1 / 2)[f(x)+f(y)] } \\
\leq & h(s+t) f(\sqrt[3]{x y z})+h(2-s-t) f(z)+h(1 / 2)[f(x)+f(y)] \\
= & h(3 / 2) f(\sqrt[3]{x y z})+h(1 / 2) f(z)+h(1 / 2)[f(x)+f(y)] \\
= & h(3 / 2) f(\sqrt[3]{x y z})+h(1 / 2)[f(x)+f(y)+f(z)],
\end{aligned}
$$

which proves the inequality (3.1).

Remark 7. In (3.1), setting $z=y$ we get

$$
2 f(\sqrt{x y})+f(y) \leq(\geq) h(3 / 2) f\left(\sqrt[3]{x y^{2}}\right)+h(1 / 2)[f(x)+2 f(y)],
$$

for all $x, y \in I$. 
Corollary 13. If $f: I \rightarrow(0, \infty)$ is $\mathrm{G}_{\mathrm{t}} \mathrm{A}_{\mathrm{t}}$-convex function, then

$$
\frac{2}{3}[f(\sqrt{x z})+f(\sqrt{y z})+f(\sqrt{x y})] \leq f(\sqrt[3]{x y z})+\frac{f(x)+f(y)+f(z)}{3},
$$

for all $x, y, z \in I$. The equality holds with $f(x)=\log (x), x>1$.

Example 10. Let $f(x)=\cosh (x), x>0$. Then, $f$ is $\mathrm{G}_{\mathrm{t}} \mathrm{A}_{\mathrm{t}}$-convex on $(0, \infty)$. Applying Corollary 31 we get

$$
\frac{2}{3}[\cosh (\sqrt{x z})+\cosh (\sqrt{y z})+\cosh (\sqrt{x y})] \leq \cosh (\sqrt[3]{x y z})+\frac{\cosh (x)+\cosh (y)+\cosh (z)}{3},
$$

for all $x, y, z>0$.

Corollary 14. If $f: I \rightarrow(0, \infty)$ is $\frac{1}{t}-\mathrm{G}_{\mathrm{t}} \mathrm{A}_{\mathrm{t}}$-concave function, then

$$
\frac{3}{2}[f(\sqrt{x z})+f(\sqrt{y z})+f(\sqrt{x y})] \geq f(\sqrt[3]{x y z})+3(f(x)+f(y)+f(z))
$$

for all $x, y, z \in I$.

Example 11. Let $f(x)=-x^{2}, x>0$. Then, $f$ is $\frac{1}{t}-\mathrm{G}_{\mathrm{t}} \mathrm{A}_{\mathrm{t}}$-concave on $(0, \infty)$. Applying Corollary 14 we get

$$
\frac{3}{2}(x z+y z+x y) \leq(\sqrt[3]{x y z})^{2}+3\left(x^{2}+y^{2}+z^{2}\right)
$$

for all $x, y, z>0$.

Corollary 15. If $f: I \rightarrow(0, \infty)$ is $1-\mathrm{G}_{\mathrm{t}} \mathrm{A}_{\mathrm{t}}$-concave function, then

$$
f(\sqrt{x z})+f(\sqrt{y z})+f(\sqrt{x y}) \geq f(\sqrt[3]{x y z})+f(x)+f(y)+f(z),
$$

for all $x, y, z \in I$.

Example 12. Let $f(x)=-x^{2}, x>0$. Then, $f$ is $1-\mathrm{G}_{\mathrm{t}} \mathrm{A}_{\mathrm{t}}$-convex on $(0, \infty)$. Applying Corollary 15 we get

$$
x z+y z+x y \leq(\sqrt[3]{x y z})^{2}+x^{2}+y^{2}+z^{2}
$$

for all $x, y, z>0$.

Corollary 16. In Theorem 5.

(1) If $f: I \rightarrow(0, \infty)$ is an $h-\mathrm{G}_{\mathrm{t}} \mathrm{A}_{\mathrm{t}}$-convex and superadditive,

$$
\begin{aligned}
f(\sqrt{x z})+f(\sqrt{y z}) & f(\sqrt{x y}) \\
\leq & h(3 / 2) f(\sqrt[3]{x y z})+h(1 / 2)[f(x)+f(y)+f(z)] \\
& \leq h(3 / 2) f(\sqrt[3]{x y z})+h(1 / 2) f(x+y+z),
\end{aligned}
$$

for all $x, y, z \in I$. If $f$ is an $h-\mathrm{G}_{\mathrm{t}} \mathrm{A}_{\mathrm{t}}$-concave and subadditive, then the inequality is reversed.

(2) If $f: I \rightarrow(0, \infty)$ is an $h-\mathrm{G}_{\mathrm{t}} \mathrm{A}_{\mathrm{t}}$-convex and subadditive, then

$$
\begin{aligned}
f(\sqrt{x z}+\sqrt{y z}+\sqrt{x y}) \leq f(\sqrt{x z})+ & f(\sqrt{y z})+f(\sqrt{x y}) \\
& \leq h(3 / 2) f(\sqrt[3]{x y z})+h(1 / 2)[f(x)+f(y)+f(z)],
\end{aligned}
$$

for all $x, y, z \in I$. If $f$ is an $h-\mathrm{G}_{\mathrm{t}} \mathrm{A}_{\mathrm{t}}$-concave and superadditive, then the inequality is reversed. 
Example 13. Let $f(x)=\cosh (x)$, which is $\mathrm{G}_{\mathrm{t}} \mathrm{A}_{\mathrm{t}}$-convex and superadditive on $(0, \infty)$. Applying Corollary 16 we get

$$
\begin{aligned}
& \frac{2}{3}[\cosh (\sqrt{x z})+\cosh (\sqrt{y z})+\cosh (\sqrt{x y})] \\
& \leq \cosh (\sqrt[3]{x y z})+\frac{\cosh (x)+\cosh (y)+\cosh (z)}{3} \\
& \quad \leq \cosh (\sqrt[3]{x y z})+\frac{1}{3} \cosh (x+y+z),
\end{aligned}
$$

for all $x, y, z>0$.

\subsection{The case when $f$ is $h-G_{\mathrm{t}} \mathrm{G}_{\mathrm{t}}$-convex.}

Theorem 6. Let $h: I \rightarrow(0, \infty)$ be a non-negative super(sub)additive function. If $f: I \rightarrow$ $(0, \infty)$ is $h-\mathrm{G}_{\mathrm{t}} \mathrm{G}_{\mathrm{t}}$-convex (-concave) function, then

$$
f(\sqrt{x z}) f(\sqrt{y z}) f(\sqrt{x y}) \leq(\geq)[f(\sqrt[3]{x y z})]^{h(3 / 2)}[f(x) f(y) f(z)]^{h(1 / 2)},
$$

for all $x, y, z \in I$.

Proof. $f$ is $h$ - $\mathrm{G}_{\mathrm{t}} \mathrm{G}_{\mathrm{t}}$-convex iff the inequality

$$
f\left(\alpha^{t} \beta^{1-t}\right) \leq[f(\alpha)]^{h(t)}[f(\beta)]^{h(1-t)}, \quad 0 \leq t \leq 1
$$

holds for all $\alpha, \beta \in I$. As in the proof of Theorem 5 , if $x y z^{2}=1$, then $x=y=z$, and Popoviciu's inequality holds.

If $s+t=\frac{3}{2}$, then since $f$ is $\mathrm{G}_{\mathrm{t}} \mathrm{G}_{\mathrm{t}}$-convex, we have

$$
\begin{aligned}
& f(\sqrt{x z})=f\left[(x y z)^{s / 3} z^{1-s}\right] \leq[f(\sqrt[3]{x y z})]^{h(s)}[f(z)]^{h(1-s)}, \\
& f(\sqrt{y z})=f\left[(x y z)^{t / 3} z^{1-t}\right] \leq[f(\sqrt[3]{x y z})]^{h(t)}[f(z)]^{h(1-t)}, \\
& f(\sqrt{x y}) \leq h\left(\frac{1}{2}\right)[f(x)+f(y)] .
\end{aligned}
$$

Multiplying these inequalities we get

$$
\begin{aligned}
& f(\sqrt{x z}) f(\sqrt{y z}) f(\sqrt{x y}) \\
& \leq[f(\sqrt[3]{x y z})]^{h(s)}[f(z)]^{h(1-s)}[f(\sqrt[3]{x y z})]^{h(t)}[f(z)]^{h(1-t)}[f(x) f(y)]^{h(1 / 2)} \\
& =[f(\sqrt[3]{x y z})]^{h(s)+h(t)}[f(z)]^{h(1-s)+h(1-t)}[f(x) f(y)]^{h(1 / 2)} \\
& \leq[f(\sqrt[3]{x y z})]^{h(s+t)}[f(z)]^{h(2-s-t)}[f(x) f(y)]^{h(1 / 2)} \\
& =[f(\sqrt[3]{x y z})]^{h(3 / 2)}[f(z)]^{h(1 / 2)}[f(x) f(y)]^{h(1 / 2)} \\
& =[f(\sqrt[3]{x y z})]^{h(3 / 2)}[f(x) f(y) f(z)]^{h(1 / 2)},
\end{aligned}
$$

which proves the inequality in (3.2).

Remark 8. In (3.2), setting $z=y$ we get

$$
f^{2}(\sqrt{x y}) f(y) \leq(\geq)\left[f\left(\sqrt[3]{x y^{2}}\right)\right]^{h(3 / 2)}\left[f(x) f^{2}(y)\right]^{h(1 / 2)},
$$

for all $x, y \in I$.

Corollary 17. If $f: I \rightarrow(0, \infty)$ is $\mathrm{G}_{\mathrm{t}} \mathrm{G}_{\mathrm{t}}$-convex (concave) function, then

$$
f^{2}(\sqrt{x z}) f^{2}(\sqrt{y z}) f^{2}(\sqrt{x y}) \leq(\geq) f^{3}(\sqrt[3]{x y z}) f(x) f(y) f(z)
$$

for all $x, y, z \in I$. The equality holds with $f(x)=\mathrm{e}^{x}, x>0$. 
Example 14. Let $f(x)=\cosh (x)$, which is $\mathrm{G}_{\mathrm{t}} \mathrm{G}_{\mathrm{t}}$-convex on $(0, \infty)$. Applying Corollary 17 we get

$$
\cosh ^{2}(\sqrt{x z}) \cosh ^{2}(\sqrt{y z}) \cosh ^{2}(\sqrt{x y}) \leq f^{3}(\sqrt[3]{x y z}) \cosh (x) \cosh (y) \cosh (z),
$$

for all $x, y, z>0$.

Corollary 18. If $f: I \rightarrow(0, \infty)$ is $\frac{1}{t}-\mathrm{G}_{\mathrm{t}} \mathrm{G}_{\mathrm{t}}$-concave function, then

$$
f^{3}(\sqrt{x z}) f^{3}(\sqrt{y z}) f^{3}(\sqrt{x y}) \geq f^{2}(\sqrt[3]{x y z}) f^{6}(x) f^{6}(y) f^{6}(z),
$$

for all $x, y, z \in I$.

Example 15. Let $f(x)=\exp (-x)$ which is $\frac{1}{t}-\mathrm{G}_{\mathrm{t}} \mathrm{G}_{\mathrm{t}}$-concave on $(0, \infty)$. Applying Corollary 18 we get

$$
\sqrt{x z}+\sqrt{y z}+\sqrt{x y} \leq \frac{2}{3} \sqrt[3]{x y z}+2 x+2 y+2 z
$$

for all $x, y, z>0$.

Corollary 19. If $f: I \rightarrow(0, \infty)$ is $1-\mathrm{G}_{\mathrm{t}} \mathrm{G}_{\mathrm{t}}$-concave function, then

$$
f(\sqrt{x z}) f(\sqrt{y z}) f(\sqrt{x y}) \leq f(\sqrt[3]{x y z}) f(x) f(y) f(z),
$$

for all $x, y, z \in I$.

Example 16. Let $f(x)=\exp (-x)$, which is $1-\mathrm{G}_{\mathrm{t}} \mathrm{G}_{\mathrm{t}}$-concave on $(0, \infty)$. Applying Corollary 19 we get

$$
\sqrt{x z}+\sqrt{y z}+\sqrt{x y} \leq \sqrt[3]{x y z}+x+y+z,
$$

for all $x, y, z>0$.

Corollary 20. In Theorem 6.

(1) If $f: I \rightarrow(0, \infty)$ is an $h-\mathrm{G}_{\mathrm{t}} \mathrm{G}_{\mathrm{t}}$-convex and supermultiplicative,

$$
\begin{aligned}
f(\sqrt{x z}) f(\sqrt{y z}) f(\sqrt{x y}) & \leq[f(\sqrt[3]{x y z})]^{h(3 / 2)}[f(x) f(y) f(z)]^{h(1 / 2)} \\
& \leq[f(\sqrt[3]{x y z})]^{h(3 / 2)}[f(x y z)]^{h(1 / 2)}
\end{aligned}
$$

for all $x, y, z \in I$.

(2) If $f: I \rightarrow(0, \infty)$ is an $h-\mathrm{G}_{\mathrm{t}} \mathrm{G}_{\mathrm{t}}$-convex and submultiplicative, then

$$
\begin{aligned}
f(x z y) & \leq f(\sqrt{x z}) f(\sqrt{y z}) f(\sqrt{x y}) \\
& \leq[f(\sqrt[3]{x y z})]^{h(3 / 2)}[f(x) f(y) f(z)]^{h(1 / 2)} \\
& \leq[f(\sqrt[3]{x}) f(\sqrt[3]{y}) f(\sqrt[3]{z})]^{h(3 / 2)}[f(x) f(y) f(z)]^{h(1 / 2)},
\end{aligned}
$$

for all $x, y, z \in I$.

Example 17. Let $f(x)=\cosh (x)$, which is $\mathrm{G}_{\mathrm{t}} \mathrm{G}_{\mathrm{t}}$-convex and supermultiplicative on $[1, \infty)$. Applying Corollary 20 we get

$$
\begin{aligned}
\cosh ^{2}(\sqrt{x z}) \cosh ^{2}(\sqrt{y z}) \cosh ^{2}(\sqrt{x y}) & \leq \cosh ^{3}(\sqrt[3]{x y z}) \cosh (x) \cosh (y) \cosh (z) \\
& \leq \cosh ^{3}(\sqrt[3]{x y z}) \cosh (x y z)
\end{aligned}
$$

for all $x, y, z \geq 1$. 


\subsection{The case when $f$ is $h-\mathrm{G}_{\mathrm{t}} \mathrm{H}_{\mathrm{t}}$-convex.}

Theorem 7. Let $h: I \rightarrow(0, \infty)$ be a non-negative super(sub)additive function. If $f: I \rightarrow$ $(0, \infty)$ is $h-\mathrm{G}_{\mathrm{t}} \mathrm{H}_{\mathrm{t}}$-concave (-convex) function, then

$$
\begin{aligned}
& \frac{1}{f(\sqrt{x z})}+\frac{1}{f(\sqrt{y z})}+\frac{1}{f(\sqrt{x y})} \\
& \leq(\geq) h\left(\frac{1}{2}\right)\left[\frac{1}{f(x)}+\frac{1}{f(y)}+\frac{1}{f(z)}\right]+\frac{h(3 / 2)}{f(\sqrt[3]{x y z})}
\end{aligned}
$$

for all $x, y, z \in I$.

Proof. $f$ is $h-\mathrm{G}_{\mathrm{t}} \mathrm{H}_{\mathrm{t}}$-convex iff the inequality

$$
f\left(\alpha^{t} \beta^{1-t}\right) \leq \frac{f(\alpha) f(\beta)}{h(1-t) f(\alpha)+h(t) f(\beta)}, \quad 0 \leq t \leq 1
$$

holds for all $\alpha, \beta \in I$. As in the proof of Theorem 5 , if $x y z^{2}=1$, then $x=y=z$, and Popoviciu's inequality holds.

If $s+t=\frac{3}{2}$, then since $f$ is $\mathrm{G}_{\mathrm{t}} \mathrm{H}_{\mathrm{t}}$-concave, we have

$$
f(\sqrt{x z})=f\left[(x y z)^{s / 3} z^{1-s}\right] \geq \frac{f(\sqrt[3]{x y z}) f(z)}{h(1-s) f(\sqrt[3]{x y z})+h(s) f(z)}
$$

and this equivalent to write

$$
\frac{1}{f(\sqrt{x z})} \leq \frac{h(1-s) f(\sqrt[3]{x y z})+h(s) f(z)}{f(\sqrt[3]{x y z}) f(z)}
$$

similarly,

$$
f(\sqrt{y z})=f\left[(x y z)^{t / 3} z^{1-t}\right] \geq \frac{f(\sqrt[3]{x y z}) f(z)}{h(1-t) f(\sqrt[3]{x y z})+h(t) f(z)}
$$

which equivalent to write

$$
\frac{1}{f(\sqrt{y z})} \leq \frac{h(1-t) f(\sqrt[3]{x y z})+h(t) f(z)}{f(\sqrt[3]{x y z}) f(z)}
$$

and

$$
\begin{aligned}
& f(\sqrt{x y}) \geq \frac{f(x) f(y)}{h(1 / 2)(f(x)+f(y))} \\
& \Longleftrightarrow \frac{1}{f(\sqrt{x y})} \leq \frac{h(1 / 2)(f(x)+f(y))}{f(x) f(y)} .
\end{aligned}
$$


Summing the inequalities (3.4)-(3.6), we get

$$
\begin{aligned}
& \frac{1}{f(\sqrt{x z})}+\frac{1}{f(\sqrt{y z})}+\frac{1}{f(\sqrt{x y})} \\
& \leq \frac{h(1-s) f(\sqrt[3]{x y z})+h(s) f(z)}{f(\sqrt[3]{x y z}) f(z)}+\frac{h(1-t) f(\sqrt[3]{x y z})+h(t) f(z)}{f(\sqrt[3]{x y z}) f(z)} \\
& \quad+\frac{h(1 / 2)(f(x)+f(y))}{f(x) f(y)} \\
& =\frac{[h(1-s)+h(1-t)] f(\sqrt[3]{x y z})+[h(s)+h(t)] f(z)}{f(\sqrt[3]{x y z}) f(z)} \\
& \quad+\frac{h(1 / 2)(f(x)+f(y))}{f(x) f(y)} \\
& \leq \frac{h(2-s-t) f(\sqrt[3]{x y z})+h(s+t) f(z)}{f(\sqrt[3]{x y z}) f(z)}+\frac{h(1 / 2)(f(x)+f(y))}{f(x) f(y)} \\
& \leq \frac{h(1 / 2) f(\sqrt[3]{x y z})+h(3 / 2) f(z)}{f(\sqrt[3]{x y z}) f(z)}+\frac{h(1 / 2)(f(x)+f(y))}{f(x) f(y)} \\
& =h\left(\frac{1}{2}\right)\left[\frac{1}{f(x)}+\frac{1}{f(y)}+\frac{1}{f(z)}\right]+\frac{h(3 / 2)}{f(\sqrt[3]{x y z})},
\end{aligned}
$$

which proves the inequality in (3.3).

Remark 9. In (3.3), setting $z=y$ then we get

$$
\frac{2}{f(\sqrt{x y})}+\frac{1}{f(y)} \leq(\geq) h\left(\frac{1}{2}\right)\left[\frac{1}{f(x)}+\frac{2}{f(y)}\right]+\frac{h(3 / 2)}{f\left(\sqrt[3]{x y^{2}}\right)}
$$

for all $x, y \in I$.

Corollary 21. If $f: I \rightarrow(0, \infty)$ is $\mathrm{G}_{\mathrm{t}} \mathrm{H}_{\mathrm{t}}$-concave (convex) function, then

$$
\frac{2}{3}\left[\frac{1}{f(\sqrt{x z})}+\frac{1}{f(\sqrt{y z})}+\frac{1}{f(\sqrt{x y})}\right] \leq(\geq) \frac{1}{3}\left[\frac{1}{f(x)}+\frac{1}{f(y)}+\frac{1}{f(z)}\right]+\frac{1}{f(\sqrt[3]{x y z})},
$$

for all $x, y, z \in I$. The equality holds with $f(x)=\frac{1}{\log (x)}, x \supsetneqq 1$.

Example 18. Let $f(x)=\cosh (x)$, then $f$ is $\mathrm{G}_{\mathrm{t}} \mathrm{H}_{\mathrm{t}}$-concave for all $x \geq 1$. Applying Corollary 21, then we get

$$
\begin{aligned}
\frac{2}{3}\left[\frac{1}{\cosh (\sqrt{x z})}+\frac{1}{\cosh (\sqrt{y z})}+\frac{1}{\cosh (\sqrt{x y})}\right] \\
\leq \frac{1}{3}\left[\frac{1}{\cosh (x)}+\frac{1}{\cosh (y)}+\frac{1}{\cosh (z)}\right]+\frac{1}{\cosh (\sqrt[3]{x y z})}
\end{aligned}
$$

for all $x, y, z \geq 1$.

Corollary 22. If $f: I \rightarrow(0, \infty)$ is $\frac{1}{t}-\mathrm{G}_{\mathrm{t}} \mathrm{H}_{\mathrm{t}}$-convex function, then

$$
\frac{3}{2}\left[\frac{1}{f(\sqrt{x z})}+\frac{1}{f(\sqrt{y z})}+\frac{1}{f(\sqrt{x y})}\right] \geq 3\left[\frac{1}{f(x)}+\frac{1}{f(y)}+\frac{1}{f(z)}\right]+\frac{1}{f(\sqrt[3]{x y z})},
$$

for all $x, y, z \in I$. 
POPOVICIU'S TYPE INEQUALITIES

Example 19. Let $f(x)=-\log (x)$, then $f$ is $\frac{1}{t}-\mathrm{G}_{\mathrm{t}} \mathrm{H}_{\mathrm{t}}$-convex for all $x>1$. Applying Corollary 22, then we get

$$
\frac{3}{2}\left[\frac{1}{\log (\sqrt{x z})}+\frac{1}{\log (\sqrt{y z})}+\frac{1}{\log (\sqrt{x y})}\right] \leq 3\left[\frac{1}{\log (x)}+\frac{1}{\log (y)}+\frac{1}{\log (z)}\right]+\frac{1}{\log (\sqrt[3]{x y z})},
$$

for all $x, y, z>1$.

Corollary 23. If $f: I \rightarrow(0, \infty)$ is $1-\mathrm{G}_{\mathrm{t}} \mathrm{H}_{\mathrm{t}}$-convex function, then

$$
\frac{1}{f(\sqrt{x z})}+\frac{1}{f(\sqrt{y z})}+\frac{1}{f(\sqrt{x y})} \geq\left[\frac{1}{f(x)}+\frac{1}{f(y)}+\frac{1}{f(z)}\right]+\frac{1}{f(\sqrt[3]{x y z})},
$$

for all $x, y, z \in I$.

Example 20. Let $f(x)=-\log (x)$, then $f$ is $1-\mathrm{G}_{\mathrm{t}} \mathrm{H}_{\mathrm{t}}$-convex for all $x>1$. Applying Corollary 23, then we get

$$
\frac{1}{\log (\sqrt{x z})}+\frac{1}{\log (\sqrt{y z})}+\frac{1}{\log (\sqrt{x y})} \leq\left[\frac{1}{\log (x)}+\frac{1}{\log (y)}+\frac{1}{\log (z)}\right]+\frac{1}{\log (\sqrt[3]{x y z})},
$$

for all $x, y, z>1$.

\section{Popoviciu inequalities for $h$ - $\mathrm{H}_{\mathrm{t}} \mathrm{N}_{\mathrm{t}}$-CONVEx functions}

In this section, we prove the corresponding version of Popoviciu inequality for the classes $h$ - $\mathrm{H}_{\mathrm{t}} \mathrm{N}_{\mathrm{t}}$-convex functions, where $\mathrm{N}_{\mathrm{t}}=\mathrm{A}_{\mathrm{t}}, \mathrm{G}_{\mathrm{t}}, \mathrm{H}_{\mathrm{t}}$.

\subsection{The case when $f$ is $h-\mathrm{H}_{\mathrm{t}} \mathrm{A}_{\mathrm{t}}$-convex.}

Theorem 8. Let $h: I \rightarrow(0, \infty)$ be a non-negative super(sub)additive. If $f: I \rightarrow(0, \infty)$ is $h-\mathrm{H}_{\mathrm{t}} \mathrm{A}_{\mathrm{t}}$-convex (concave) function, then

$$
\begin{aligned}
& f\left(\frac{2 x z}{x+z}\right)+f\left(\frac{2 y z}{y+z}\right)+f\left(\frac{2 x y}{x+y}\right) \\
& \leq(\geq) h(3 / 2) f\left(\frac{3 x y z}{x y+y z+x z}\right)+h(1 / 2)[f(x)+f(y)+f(z)],
\end{aligned}
$$

for all $x, y, z \in I$.

Proof. $f$ is $h-\mathrm{H}_{\mathrm{t}} \mathrm{A}_{\mathrm{t}}$-convex iff the inequality

$$
f\left(\frac{\alpha \beta}{t \alpha+(1-t) \beta}\right) \leq h(1-t) f(\alpha)+h(t) f(\beta), \quad 0 \leq t \leq 1,
$$

holds for all $\alpha, \beta \in I$. Assume that $x \leq y \leq z$. If $y \leq \frac{3 x y z}{x y+y z+x z}$, then

$$
\frac{3 x y z}{x y+y z+x z} \leq \frac{2 x z}{x+z} \leq z \text { and } \frac{3 x y z}{x y+y z+x z} \leq \frac{2 y z}{y+z} \leq z,
$$

so that there exist two numbers $s, t \in[0,1]$ satisfying

$$
\frac{2 x z}{x+z}=\frac{\frac{3 x y z}{x y+y z+x z} \cdot z}{s \frac{3 x y z}{x y+y z+x z}+(1-s) z},
$$

and

$$
\frac{2 y z}{y+z}=\frac{\frac{3 x y z}{x y+y z+x z} \cdot z}{t \frac{3 x y z}{x y+y z+x z}+(1-t) z} .
$$


For simplicity set, $u=\frac{3 x y z}{x y+y z+x z}$, summing the reciprocal of the previous two equations

$$
\frac{x+z}{2 x z}+\frac{y+z}{2 y z}=\frac{(s+t) \frac{3 x y z}{x y+y z+x z}+(2-s-t) z}{\frac{3 x y z}{x y+y z+x z} \cdot z}=\frac{3(s+t) u+(2-s-t) z}{3 u \cdot z} .
$$

Simplifying the above equation and reverse it back to the original form (taking the reciprocal again), we get

$$
\frac{u}{u+z}=\frac{u}{2(s+t) u+\frac{2}{3}(2-s-t) z},
$$

since $y, x, z>0$, this yields that $x=y=z$ and thus Popoviciu's inequality holds, or $s+t=\frac{1}{2}$ and in this case since $f$ is $\mathrm{H}_{\mathrm{t}} \mathrm{A}_{\mathrm{t}}$-convex, we have

$$
\begin{aligned}
& f\left(\frac{2 x z}{x+z}\right)=f\left(\frac{\frac{3 x y z}{x y+y z+x z} \cdot z}{s \frac{3 x y z}{x y+y z+x z}+(1-s) z}\right) \leq h(s) f(z)+h(1-s) f\left(\frac{3 x y z}{x y+y z+x z}\right), \\
& f\left(\frac{2 y z}{y+z}\right)=f\left(\frac{\frac{3 x y z}{x y+y z+x z} \cdot z}{t \frac{3 x y z}{x y+y z+x z}+(1-t) z}\right) \leq h(t) f(z)+h(1-t) f\left(\frac{3 x y z}{x y+y z+x z}\right), \\
& f\left(\frac{2 x y}{x+y}\right) \leq h(1 / 2)[f(x)+f(y)] .
\end{aligned}
$$

Summing up these inequalities we get

$$
\begin{aligned}
& f\left(\frac{2 x z}{x+z}\right)+f\left(\frac{2 y z}{y+z}\right)+f\left(\frac{2 x y}{x+y}\right) \\
& \leq[h(s)+h(t)] f(z)+[h(1-s)+h(1-t)] f\left(\frac{3 x y z}{x y+y z+x z}\right)+h(1 / 2)[f(x)+f(y)] \\
& \leq h(s+t) f(z)+h(2-s-t) f\left(\frac{3 x y z}{x y+y z+x z}\right)+h(1 / 2)[f(x)+f(y)] \\
& =h(3 / 2) f\left(\frac{3 x y z}{x y+y z+x z}\right)+h(1 / 2)[f(x)+f(y)+f(z)],
\end{aligned}
$$

which proves the inequality in (4.1).

Remark 10. In (4.1), setting $z=y$ then we get

$$
2 f\left(\frac{2 x y}{x+y}\right)+f(y) \leq(\geq) h(3 / 2) f\left(\frac{3 x y}{2 x+y}\right)+h(1 / 2)[f(x)+2 f(y)],
$$

for all $x, y \in I$.

Corollary 24. If $f: I \rightarrow(0, \infty)$ is $\mathrm{H}_{\mathrm{t}} \mathrm{A}_{\mathrm{t}}$-convex (concave) function, then

$$
\begin{aligned}
\frac{2}{3}\left[f\left(\frac{2 x z}{x+z}\right)+f\left(\frac{2 y z}{y+z}\right)+f\left(\frac{2 x y}{x+y}\right)\right] & \\
& \leq(\geq) f\left(\frac{3 x y z}{x y+y z+x z}\right)+\frac{f(x)+f(y)+f(z)}{3}
\end{aligned}
$$

for all $x, y, z \in I$. The equality holds with $f(x)=\frac{1}{x}, x>0$. 
Example 21. Let $f(x)=\arctan (x)$, then $f$ is $\mathrm{H}_{\mathrm{t}} \mathrm{A}_{\mathrm{t}}$-convex on $(0, \infty)$. Applying Corollary 24, then we get

$$
\begin{aligned}
\frac{2}{3}\left[\arctan \left(\frac{2 x z}{x+z}\right)+\arctan \left(\frac{2 y z}{y+z}\right)+\arctan \left(\frac{2 x y}{x+y}\right)\right] \\
\leq \arctan \left(\frac{3 x y z}{x y+y z+x z}\right)+\frac{\arctan (x)+\arctan (y)+\arctan (z)}{3}
\end{aligned}
$$

Corollary 25. If $f: I \rightarrow(0, \infty)$ is $\frac{1}{t}-\mathrm{H}_{\mathrm{t}} \mathrm{A}_{\mathrm{t}}$-concave function, then

$$
\frac{3}{2}\left[f\left(\frac{2 x z}{x+z}\right)+f\left(\frac{2 y z}{y+z}\right)+f\left(\frac{2 x y}{x+y}\right)\right] \geq f\left(\frac{3 x y z}{x y+y z+x z}\right)+3[f(x)+f(y)+f(z)],
$$

for all $x, y, z \in I$.

Example 22. Let $f(x)=-x^{2}$, then $f$ is $\frac{1}{t}-\mathrm{H}_{\mathrm{t}} \mathrm{A}_{\mathrm{t}}$-concave on $x>0$. Applying Corollary 25, then we get

$$
\left(\frac{x z}{x+z}\right)^{2}+\left(\frac{y z}{y+z}\right)^{2}+\left(\frac{x y}{x+y}\right)^{2} \leq \frac{3}{2}\left(\frac{x y z}{x y+y z+x z}\right)^{2}+\frac{1}{18}\left(x^{2}+y^{2}+z^{2}\right),
$$

for all $x, y, z<0$.

Corollary 26. If $f: I \rightarrow(0, \infty)$ is $1-\mathrm{H}_{\mathrm{t}} \mathrm{A}_{\mathrm{t}}$-concave function, then

$$
f\left(\frac{2 x z}{x+z}\right)+f\left(\frac{2 y z}{y+z}\right)+f\left(\frac{2 x y}{x+y}\right) \geq f\left(\frac{3 x y z}{x y+y z+x z}\right)+[f(x)+f(y)+f(z)],
$$

for all $x, y, z \in I$.

Example 23. Let $f(x)=-x^{2}$, then $f$ is $1-\mathrm{H}_{\mathrm{t}} \mathrm{A}_{\mathrm{t}}$-concave on $(0, \infty)$. Applying Corollary 26, then we get

$$
\left(\frac{x z}{x+z}\right)^{2}+\left(\frac{y z}{y+z}\right)^{2}+\left(\frac{x y}{x+y}\right)^{2} \leq \frac{9}{4}\left[\frac{x^{2}+y^{2}+z^{2}}{9}+\left(\frac{x y z}{x y+y z+x z}\right)^{2}\right]
$$

for all $x, y, z<0$.

\section{Corollary 27. In Theorem 8.}

(1) If $f: I \rightarrow(0, \infty)$ is an $h-\mathrm{H}_{\mathrm{t}} \mathrm{A}_{\mathrm{t}}$-convex and superadditive, then

$$
\begin{aligned}
& 2\left[f\left(\frac{x z}{x+z}\right)+f\left(\frac{y z}{y+z}\right)+f\left(\frac{x y}{x+y}\right)\right] \\
& \leq f\left(\frac{2 x z}{x+z}\right)+f\left(\frac{2 y z}{y+z}\right)+f\left(\frac{2 x y}{x+y}\right) \\
& \leq h(3 / 2) f\left(\frac{3 x y z}{x y+y z+x z}\right)+h(1 / 2)[f(x)+f(y)+f(z)] \\
& \leq h(3 / 2) f\left(\frac{3 x y z}{x y+y z+x z}\right)+h(1 / 2) f(x+y+z),
\end{aligned}
$$

for all $x, y, z \in I$. If $f$ is an $h-\mathrm{H}_{\mathrm{t}} \mathrm{A}_{\mathrm{t}}$-concave and subadditive, then the inequality is reversed. 
(2) If $f: I \rightarrow(0, \infty)$ is an $h-\mathrm{H}_{\mathrm{t}} \mathrm{A}_{\mathrm{t}}$-convex and subadditive, then

$$
\begin{aligned}
& f\left(\frac{2 x z}{x+z}+\frac{2 y z}{y+z}+\frac{2 x y}{x+y}\right) \\
& \leq f\left(\frac{2 x z}{x+z}\right)+f\left(\frac{2 y z}{y+z}\right)+f\left(\frac{2 x y}{x+y}\right) \\
& \leq h(3 / 2) f\left(\frac{3 x y z}{x y+y z+x z}\right)+h(1 / 2)[f(x)+f(y)+f(z)] \\
& \leq 3 h(3 / 2) f\left(\frac{x y z}{x y+y z+x z}\right)+h(1 / 2)[f(x)+f(y)+f(z)],
\end{aligned}
$$

for all $x, y, z \in I$. If $f$ is an $h-\mathrm{H}_{\mathrm{t}} \mathrm{A}_{\mathrm{t}}$-concave and superadditive, then the inequality is reversed.

\subsection{The case when $f$ is $h-\mathrm{H}_{\mathrm{t}} \mathrm{G}_{\mathrm{t}}$-convex.}

Theorem 9. Let $h: I \rightarrow(0, \infty)$ be a non-negative super(sub)additive. If $f: I \rightarrow(0, \infty)$ is $h-\mathrm{H}_{\mathrm{t}} \mathrm{G}_{\mathrm{t}}$-convex (-concave) function, then

$$
\begin{aligned}
& f\left(\frac{2 x z}{x+z}\right)+f\left(\frac{2 y z}{y+z}\right)+f\left(\frac{2 x y}{x+y}\right) \\
& \leq(\geq)\left[f\left(\frac{3 x y z}{x y+y z+x z}\right)\right]^{h(3 / 2)}[f(x) f(y) f(z)]^{h(1 / 2)}
\end{aligned}
$$

for all $x, y, z \in I$.

Proof. $f$ is $h-\mathrm{H}_{\mathrm{t}} \mathrm{G}_{\mathrm{t}}$-convex iff the inequality

$$
f\left(\frac{\alpha \beta}{t \alpha+(1-t) \beta}\right) \leq[f(\alpha)]^{h(1-t)}[f(\beta)]^{h(t)}, \quad 0 \leq t \leq 1 .
$$

holds for all $\alpha, \beta \in I$. As in the proof of Theorem 8 , if $x=y=z$, then the inequality holds. If $s+t=\frac{1}{2}$ since $f$ is $\mathrm{H}_{\mathrm{t}} \mathrm{G}_{\mathrm{t}}$-convex, we have

$$
\begin{aligned}
& f\left(\frac{2 x z}{x+z}\right)=f\left(\frac{\frac{3 x y z}{x y+y z+x z} \cdot z}{s \frac{3 x y z}{x y+y z+x z}+(1-s) z}\right) \leq[f(z)]^{h(s)}\left[f\left(\frac{3 x y z}{x y+y z+x z}\right)\right]^{h(1-s)}, \\
& f\left(\frac{2 y z}{y+z}\right)=f\left(\frac{\frac{3 x y z}{x y+y z+x z} \cdot z}{t \frac{3 x y z}{x y+y z+x z}+(1-t) z}\right) \leq[f(z)]^{h(t)}\left[f\left(\frac{3 x y z}{x y+y z+x z}\right)\right]^{h(1-t)}, \\
& f\left(\frac{2 x y}{x+y}\right) \leq[f(x) f(y)]^{h(1 / 2)} .
\end{aligned}
$$


Multiplying these inequalities we get

$$
\begin{aligned}
& f\left(\frac{2 x z}{x+z}\right) f\left(\frac{2 y z}{y+z}\right) f\left(\frac{2 x y}{x+y}\right) \\
& \leq[f(z)]^{h(s)}\left[f\left(\frac{3 x y z}{x y+y z+x z}\right)\right]^{h(1-s)}[f(z)]^{h(t)}\left[f\left(\frac{3 x y z}{x y+y z+x z}\right)\right]^{h(1-t)}[f(x) f(y)]^{h(1 / 2)} \\
& \leq[f(z)]^{h(s)+h(t)}\left[f\left(\frac{3 x y z}{x y+y z+x z}\right)\right]^{h(1-s)+h(1-t)}[f(x) f(y)]^{h(1 / 2)} \\
& \leq[f(z)]^{h(s+t)}\left[f\left(\frac{3 x y z}{x y+y z+x z}\right)\right]^{h(2-s-t)}[f(x) f(y)]^{h(1 / 2)} \\
& =[f(z)]^{h(1 / 2)}\left[f\left(\frac{3 x y z}{x y+y z+x z}\right)\right]^{h(3 / 2)}[f(x) f(y)]^{h(1 / 2)} \\
& =\left[f\left(\frac{3 x y z}{x y+y z+x z}\right)\right]^{h(3 / 2)}[f(x) f(y) f(z)]^{h(1 / 2)}
\end{aligned}
$$

which proves the inequality in (4.2).

Remark 11. In (4.2), setting $z=y$ we get that

$$
2 f\left(\frac{2 x y}{x+y}\right) f(y) \leq(\geq)\left[f\left(\frac{3 x y}{2 x+y}\right)\right]^{h(3 / 2)}\left[f(x) f^{2}(y)\right]^{h(1 / 2)}
$$

for all $x, y \in I$.

Corollary 28. If $f: I \rightarrow(0, \infty)$ is $\mathrm{H}_{\mathrm{t}} \mathrm{G}_{\mathrm{t}}$-convex (concave) function, then

$$
\begin{aligned}
& f\left(\frac{2 x z}{x+z}\right) f\left(\frac{2 y z}{y+z}\right) f\left(\frac{2 x y}{x+y}\right) \\
& \leq(\geq)\left[f\left(\frac{3 x y z}{x y+y z+x z}\right)\right]^{3 / 2}[f(x) f(y) f(z)]^{1 / 2}
\end{aligned}
$$

for all $x, y, z \in I$. The equality holds with $f(x)=\mathrm{e}^{\frac{1}{x}}, x>0$.

Example 24. Let $f(x)=\exp (x), x>0$. Then, $f$ is $\mathrm{H}_{\mathrm{t}} \mathrm{G}_{\mathrm{t}}$-convex on $(0, \infty)$. Applying Corollary 28 we get

$$
\frac{4 x z}{x+z}+\frac{4 y z}{y+z}+\frac{4 x y}{x+y} \leq \frac{9 x y z}{x y+y z+x z}+x y z
$$

for all $x, y, z>0$.

Corollary 29. If $f: I \rightarrow(0, \infty)$ is $\frac{1}{t}-\mathrm{H}_{\mathrm{t}} \mathrm{G}_{\mathrm{t}}$-concave, then

$$
\begin{aligned}
& f\left(\frac{2 x z}{x+z}\right) f\left(\frac{2 y z}{y+z}\right) f\left(\frac{2 x y}{x+y}\right) \\
& \geq\left[f\left(\frac{3 x y z}{x y+y z+x z}\right)\right]^{2 / 3}[f(x) f(y) f(z)]^{2},
\end{aligned}
$$

for all $x, y, z \in I$.

Example 25. Let $f(x)=\exp (-x), x>0$. Then, $f$ is $\frac{1}{t}-\mathrm{H}_{\mathrm{t}} \mathrm{G}_{\mathrm{t}}$-concave on $(0, \infty)$. Applying Corollary 29 we get

$$
\frac{x z}{x+z}+\frac{y z}{y+z}+\frac{x y}{x+y} \leq \frac{x y z}{x y+y z+x z}+x y z
$$


for all $x, y, z>0$.

Corollary 30. If $f: I \rightarrow(0, \infty)$ is $1-\mathrm{H}_{\mathrm{t}} \mathrm{G}_{\mathrm{t}}$-concave function, then

for all $x, y, z \in I$.

$$
\begin{aligned}
& f\left(\frac{2 x z}{x+z}\right) f\left(\frac{2 y z}{y+z}\right) f\left(\frac{2 x y}{x+y}\right) \\
& \geq f\left(\frac{3 x y z}{x y+y z+x z}\right) f(x) f(y) f(z),
\end{aligned}
$$

Example 26. Let $f(x)=\exp (-x), x>0$. Then, $f$ is $1-\mathrm{H}_{\mathrm{t}} \mathrm{G}_{\mathrm{t}}$-concave on $(0, \infty)$. Applying Corollary 30 we get

$$
\frac{2 x z}{x+z}+\frac{2 y z}{y+z}+\frac{2 x y}{x+y} \leq \frac{3 x y z}{x y+y z+x z}+x+y+z
$$

for all $x, y, z>0$.

Corollary 31. In Theorem 9.

(1) If $f: I \rightarrow(0, \infty)$ is an $h-\mathrm{H}_{\mathrm{t}} \mathrm{G}_{\mathrm{t}}$-convex and superadditive, then

$$
\begin{aligned}
& 2\left[f\left(\frac{x z}{x+z}\right)+f\left(\frac{y z}{y+z}\right)+f\left(\frac{x y}{x+y}\right)\right] \\
& \leq f\left(\frac{2 x z}{x+z}\right)+f\left(\frac{2 y z}{y+z}\right)+f\left(\frac{2 x y}{x+y}\right) \\
& \leq\left[f\left(\frac{3 x y z}{x y+y z+x z}\right)\right]^{h(3 / 2)}[f(x) f(y) f(z)]^{h(1 / 2)},
\end{aligned}
$$

for all $x, y, z \in I$. If $f$ is an $h-\mathrm{H}_{\mathrm{t}} \mathrm{G}_{\mathrm{t}}$-concave and subadditive, then the inequality is reversed.

(2) If $f: I \rightarrow(0, \infty)$ is an $h-\mathrm{H}_{\mathrm{t}} \mathrm{G}_{\mathrm{t}}$-convex and subadditive, then

$$
\begin{aligned}
& f\left(\frac{2 x z}{x+z}+\frac{2 y z}{y+z}+\frac{2 x y}{x+y}\right) \\
& \leq f\left(\frac{2 x z}{x+z}\right)+f\left(\frac{2 y z}{y+z}\right)+f\left(\frac{2 x y}{x+y}\right) \\
& \leq\left[f\left(\frac{3 x y z}{x y+y z+x z}\right)\right]^{h(3 / 2)}[f(x) f(y) f(z)]^{h(1 / 2)} \\
& \leq\left[3 f\left(\frac{x y z}{x y+y z+x z}\right)\right]^{h(3 / 2)}[f(x) f(y) f(z)]^{h(1 / 2)},
\end{aligned}
$$

for all $x, y, z \in I$. If $f$ is an $h-\mathrm{H}_{\mathrm{t}} \mathrm{G}_{\mathrm{t}}$-concave and superadditive, then the inequality is reversed.

\subsection{The case when $f$ is $h-\mathrm{H}_{\mathrm{t}} \mathrm{H}_{\mathrm{t}}$-convex.}

Theorem 10. Let $h: I \rightarrow(0, \infty)$ be a non-negative super(sub)additive. If $f: I \rightarrow(0, \infty)$ is $h$ - $\mathrm{H}_{\mathrm{t}} \mathrm{H}_{\mathrm{t}}$-concave (-convex) function, then

$$
\begin{aligned}
& \frac{1}{f\left(\frac{2 x z}{x+z}\right)}+\frac{1}{f\left(\frac{2 y z}{y+z}\right)}+\frac{1}{f\left(\frac{2 x y}{x+y}\right)} \\
& \leq(\geq) h\left(\frac{1}{2}\right)\left[\frac{1}{f(x)}+\frac{1}{f(y)}+\frac{1}{f(z)}\right]+\frac{h(3 / 2)}{f\left(\frac{3 x y z}{x y+y z+x z}\right)}
\end{aligned}
$$


for all $x, y, z \in I$.

Proof. $f$ is $\mathrm{H}_{\mathrm{t}} \mathrm{H}_{\mathrm{t}}$-convex iff the inequality

$$
f\left(\frac{\alpha \beta}{t \alpha+(1-t) \beta}\right) \leq \frac{f(\alpha) f(\beta)}{h(t) f(\alpha)+h(1-t) f(\beta)}, \quad 0 \leq t \leq 1
$$

holds for all $\alpha, \beta \in I$. As in the proof of Theorem 8 , if $x=y=z$, then the inequality holds. If $s+t=\frac{1}{2}$ since $f$ is $\mathrm{H}_{\mathrm{t}} \mathrm{H}_{\mathrm{t}}$-concave, we have

$$
\begin{aligned}
& f\left(\frac{2 x z}{x+z}\right)=f\left(\frac{\frac{3 x y z}{x y+y z+x z} \cdot z}{s \frac{3 x y z}{x y+y z+x z}+(1-s) z}\right) \geq \frac{f\left(\frac{3 x y z}{x y+y z+x z}\right) \cdot f(z)}{h(s) f\left(\frac{3 x y z}{x y+y z+x z}\right)+h(1-s) f(z)} \\
& f\left(\frac{2 y z}{y+z}\right)=f\left(\frac{\frac{3 x y z}{x y+y z+x z} \cdot z}{t \frac{3 x y z}{x y+y z+x z}+(1-t) z}\right) \geq \frac{f\left(\frac{3 x y z}{x y+y z+x z}\right) \cdot f(z)}{h(t) f\left(\frac{3 x y z}{x y+y z+x z}\right)+h(1-t) f(z)}, \\
& f\left(\frac{2 x y}{x+y}\right) \geq \frac{f(x) f(y)}{h(1 / 2)[f(x)+f(y)]}
\end{aligned}
$$

Therefore, by summing the reciprocal of the above inequalities we get

$$
\begin{aligned}
& \frac{1}{f\left(\frac{2 x z}{x+z}\right)}+\frac{1}{f\left(\frac{2 y z}{y+z}\right)}+\frac{1}{f\left(\frac{2 x y}{x+y}\right)} \\
\leq & \frac{h(s) f\left(\frac{3 x y z}{x y+y z+x z}\right)+h(1-s) f(z)+h(t) f\left(\frac{3 x y z}{x y+y z+x z}\right)+h(1-t) f(z)}{f\left(\frac{3 x y z}{x y+y z+x z}\right) \cdot f(z)} \\
& \quad+\frac{h(1 / 2)[f(x)+f(y)]}{f(x) f(y)} \\
\leq & \frac{[h(s)+h(s)] f\left(\frac{3 x y z}{x y+y z+x z}\right)+[h(1-s)+h(1-t)] f(z)}{f\left(\frac{3 x y z}{x y+y z+x z}\right) \cdot f(z)}+\frac{h(1 / 2)[f(x)+f(y)]}{f(x) f(y)} \\
\leq & \frac{h(s+t) f\left(\frac{3 x y z}{x y+y z+x z}\right)+h(2-s-t) f(z)}{f\left(\frac{3 x y z}{x y+y z+x z}\right) \cdot f(z)}+\frac{h(1 / 2)[f(x)+f(y)]}{f(x) f(y)} \\
= & \frac{h(1 / 2) f\left(\frac{3 x y z}{x y+y z+x z}\right)+h(3 / 2) f(z)}{f\left(\frac{3 x y z}{x y+y z+x z}\right) \cdot f(z)}+\frac{h(1 / 2)[f(x)+f(y)]}{f(x) f(y)} \\
= & \frac{h(1 / 2) f\left(\frac{3 x y z}{x y+y z+x z}\right)+h(3 / 2) f(z)}{f\left(\frac{3 x y z}{x y+y z+x z}\right) \cdot f(z)}+\frac{h(1 / 2)[f(x)+f(y)]}{f(x) f(y)} \\
= & h\left(\frac{1}{2}\right)\left[\frac{1}{f(x)}+\frac{1}{f(y)}+\frac{1}{f(z)}\right]+\frac{h(3 / 2)}{f\left(\frac{3 x y z}{x y+y z+x z}\right)},
\end{aligned}
$$

which proves the inequality in (4.3). 
Remark 12. In (4.3), setting $z=y$ then we get

$$
\frac{2}{f\left(\frac{2 x y}{x+y}\right)}+\frac{1}{f(y)} \leq(\geq) h\left(\frac{1}{2}\right)\left[\frac{1}{f(x)}+\frac{2}{f(y)}\right]+\frac{h(3 / 2)}{f\left(\frac{3 x y}{2 x+y}\right)}
$$

for all $x, y, z \in I$.

Corollary 32. If $f: I \rightarrow(0, \infty)$ is $\mathrm{H}_{\mathrm{t}} \mathrm{H}_{\mathrm{t}}$-concave (convex) function, then

$$
\begin{aligned}
& \frac{2}{3}\left[\frac{1}{f\left(\frac{2 x z}{x+z}\right)}+\frac{1}{f\left(\frac{2 y z}{y+z}\right)}+\frac{1}{f\left(\frac{2 x y}{x+y}\right)}\right] \\
& \leq(\geq) \frac{1}{3}\left[\frac{1}{f(x)}+\frac{1}{f(y)}+\frac{1}{f(z)}\right]+\frac{1}{f\left(\frac{3 x y z}{x y+y z+x z}\right)},
\end{aligned}
$$

for all $x, y, z \in I$. The equality holds with $f(x)=x, x>1$.

Example 27. Let $f(x)=\arctan (x), x>0$. Then $f$ is $\mathrm{H}_{\mathrm{t}} \mathrm{H}_{\mathrm{t}}$-concave on $(0, \infty)$. Applying Corollary 32, then we get

$$
\begin{aligned}
& \frac{2}{3}\left[\frac{1}{\arctan \left(\frac{2 x z}{x+z}\right)}+\frac{1}{\arctan \left(\frac{2 y z}{y+z}\right)}+\frac{1}{\arctan \left(\frac{2 x y}{x+y}\right)}\right] \\
& \leq \frac{1}{3}\left[\frac{1}{\arctan (x)}+\frac{1}{\arctan (y)}+\frac{1}{\arctan (z)}\right]+\frac{1}{\arctan \left(\frac{3 x y z}{x y+y z+x z}\right)},
\end{aligned}
$$

for all $x, y, z>0$.

Corollary 33. If $f: I \rightarrow(0, \infty)$ is $\frac{1}{t}-\mathrm{H}_{\mathrm{t}} \mathrm{H}_{\mathrm{t}}$-convex function, then

$$
\begin{aligned}
& \frac{3}{2}\left[\frac{1}{f\left(\frac{2 x z}{x+z}\right)}+\frac{1}{f\left(\frac{2 y z}{y+z}\right)}+\frac{1}{f\left(\frac{2 x y}{x+y}\right)}\right] \\
& \geq 3\left[\frac{1}{f(x)}+\frac{1}{f(y)}+\frac{1}{f(z)}\right]+\frac{1}{f\left(\frac{3 x y z}{x y+y z+x z}\right)},
\end{aligned}
$$

for all $x, y, z \in I$.

Example 28. Let $f(x)=-\log (x), x>1$. Then $f$ is $\frac{1}{t}-\mathrm{H}_{\mathrm{t}} \mathrm{H}_{\mathrm{t}}$-convex on $(0, \infty)$. Applying Corollary 33, then we get

$$
\begin{aligned}
& \frac{3}{2}\left[\frac{1}{\log \left(\frac{2 x z}{x+z}\right)}+\frac{1}{\log \left(\frac{2 y z}{y+z}\right)}+\frac{1}{\log \left(\frac{2 x y}{x+y}\right)}\right] \\
& \leq 3\left[\frac{1}{\log (x)}+\frac{1}{\log (y)}+\frac{1}{\log (z)}\right]+\frac{1}{\log \left(\frac{3 x y z}{x y+y z+x z}\right)},
\end{aligned}
$$

for all $x, y, z>0$. 
Corollary 34. If $f: I \rightarrow(0, \infty)$ is $1-\mathrm{H}_{\mathrm{t}} \mathrm{H}_{\mathrm{t}}$-convex function, then

$$
\begin{aligned}
& \frac{1}{f\left(\frac{2 x z}{x+z}\right)}+\frac{1}{f\left(\frac{2 y z}{y+z}\right)}+\frac{1}{f\left(\frac{2 x y}{x+y}\right)} \\
& \geq\left[\frac{1}{f(x)}+\frac{1}{f(y)}+\frac{1}{f(z)}\right]+\frac{1}{f\left(\frac{3 x y z}{x y+y z+x z}\right)},
\end{aligned}
$$

for all $x, y, z \in I$.

Example 29. Let $f(x)=-\log (x), x>0$. Then $f$ is $1-\mathrm{H}_{\mathrm{t}} \mathrm{H}_{\mathrm{t}}$-convex on $(0, \infty)$. Applying Corollary 34, then we get

$$
\begin{aligned}
& \frac{1}{\log \left(\frac{2 x z}{x+z}\right)}+\frac{1}{\log \left(\frac{2 y z}{y+z}\right)}+\frac{1}{\log \left(\frac{2 x y}{x+y}\right)} \\
& \leq\left[\frac{1}{\log (x)}+\frac{1}{\log (y)}+\frac{1}{\log (z)}\right]+\frac{1}{\log \left(\frac{3 x y z}{x y+y z+x z}\right)},
\end{aligned}
$$

for all $x, y, z>0$.

\section{REFERENCES}

[1] M.W. Alomari, Some properties of $h$-MN-convexity and Jensen's type inequalities, Preprint (2017), avaliable at arXiv: $1710.03418 \mathrm{v} 3$.

[2] G. D. Anderson, M. K. Vamanamurthy and M. Vuorinen, Generalized convexity and inequalities, J. Math. Anal. Appl., 335 (2007), 1294-1308.

[3] M. Bencze, C.P. Niculescu and F. Popovici, Popovicius inequality for functions of several variables, J. Math. Anal. Appl., 365 (2010), 399-409.

[4] W.W. Breckner, Stetigkeitsaussagen für eine Klasse verallgemeinerter konvexer funktionen in topologischen linearen Räumen, Publ. Inst. Math., 23 (1978), 13-20.

[5] S.S. Dragomir, J. Pečarić and L.E. Persson, Some inequalities of Hadamard type, Soochow J. Math., 21 (1995) 335-341.

[6] W. Fechner, Hlawka's functional inequality, Aequat. Math., 87 (2014), 71-87.

[7] E.K. Godunova and V.I. Levin, Neravenstva dlja funkcii širokogo klassa, soderžaščego vypuklye, monotonnye i nekotorye drugie vidy funkcii, Vyčislitel. Mat. i. Mat. Fiz. Mevuzov. Sb. Nauc. Trudov, MGPI, Moskva, 1985, 138-142.

[8] H. Hudzik and L. Maligranda, Some remarks on s-convex functions, Aequationes Math., 48 (1994), $100-111$.

[9] J. Jensen, Sur les fonctions convexes et les inégalités entre les valeurs moyennes, Acta Math., 30 (1906), $175-193$.

[10] D.S. Mitrinović and J. Pečarić, Note on a class of functions of Godunova and Levin, C. R. Math. Rep. Acad. Sci. Can., 12 (1990), 33-36.

[11] D.S. Mitrinović, J. Pečarić and A.M. Fink, Classical and New Inequalities in Analysis, Kluwer Academic, Dordrecht, 1993.

[12] C.P. Niculescu, Convexity according to the geometric mean, Math. Inequal. Appl., 3 (2) (2000), 155-167.

[13] C.P. Niculescu, L.E. Persson, Convex Functions and Their Applications. A Contemporary Approach, CMS Books Math., vol. 23, Springer-Verlag, New York, 2006.

[14] C.P. Niculescu, F. Popoviciu, A refinement of Popoviciu's inequality, Bull. Math. Soc. Sci. Math. Roumanie, Tome 49 (3) (2006), 285-290.

[15] C.E.M. Pearce and A.M. Rubinov, $P$-functions, quasi-convex functions and Hadamard-type inequalities, $J$. Math. Anal. Appl., 240 (1999), 92-104.

[16] T. Popoviciu, Sur certaines inégalités qui caractérisent les fonctions convexes, Analele Stiintifice Univ. Al. I. Cuza, Iasi, Sectia Mat., 11 (1965), 155-164. 
[17] D.M. Smiley and M.F. Smiley, The polygonal inequalities, Amer. Math. Mon., 71 (7) (1964), 755-760.

[18] S. Varošanec, On h-convexity, J. Math. Anal. Appl., 326 (2007), 303-11.

[19] P.M. Vasić and L.R. Stanković, Some inequalities for convex functions, Math. Balkanica, 6 (1976), 281-288.

[20] R. Whitty, A generalised Hlawka inequality by D. Smiley \& M. Smiley, Theorem of The day, availiable at: http://www. theoremoftheday.org/Analysis/Hlawka/TotDHlawka.pdf

Department of Mathematics, Faculty of Science and Information Technology, Irbid National University, 2600 Irbid 21110, Jordan.

E-mail address: mwomath@gmail.com 